\title{
Triple-point shear layers in gaseous detonation waves
}

\author{
L. MASSA ${ }^{1}$, J. M. AUSTIN ${ }^{2}$ AND T. L. JACKSON \\ ${ }^{1}$ Center for Simulation of Advanced Rockets, University of Illinois Urbana-Champaign, \\ Urbana, IL, USA \\ ${ }^{2}$ Department of Aerospace Engineering, University of Illinois Urbana-Champaign, Urbana, IL, USA
}

(Received 21 July 2006 and in revised form 16 April 2007)

Recent experiments have shown intriguing regions of intense luminescence or 'hotspots' in the vicinity of triple-point shear layers in propagating gaseous detonation waves. Localized explosions have also been observed to develop in these fronts. These features were observed in higher effective activation energy mixtures, but not in lower effective activation energy mixtures. The increased lead shock oscillation through a cell cycle in higher activation energy mixtures may result in a significantly increased disparity in the induction time on either side of the triple-point shear layer, and thus an enhanced mixing between reacted and non-reacted streams supported by Kelvin-Helmholtz instability. The relation between the shear-layer instability and the mixture effective activation energy is analysed by carrying out a spatial linear stability study for three mixtures with different activation energies and injection conditions that correspond to the experimental conditions. The role of vortical structures associated with Kelvin-Helmholtz instability in the formation of localized ignition is investigated by performing two-dimensional Navier-Stokes simulations with detailed chemical kinetics and transport. In the low activation energy mixture, large-scale vortical structures are observed to occur downstream of the induction distance; these structures do not have a noticeable effect on the reaction. In higher effective activation energy mixtures, a thin transverse ignition front develops near the interface between the two gas streams and results in a combustion structure decoupled from the entrainment region. The decoupling leads to attenuation of the instability growth rate when compared to frozen calculations, and a reduced heat release in the high vorticity region. The analysis indicates the instability plays a modest role in ignition events for high activation energy mixtures. The formation of localized explosions observed in high activation energy systems is instead linked to the impossibility of a one-dimensional reactive combustion wave supported by the injection conditions. In the absence of curvature effects and stream-tube divergence, a system of shock waves is formed which spreads the ignition to the cold gas stream.

\section{Introduction}

A detonation front propagating through a premixed combustible gas can be modelled as a one-dimensional steady wave in which the dominant combustion mechanism is shock-induced chemical-thermal branching. However, the reaction rate behind the shock is extremely sensitive to perturbations in the post-shock temperature and as a result gaseous detonation waves are always unstable (Erpenbeck 1964; Lee \& Stewart 1990; Short \& Quirk 1997; Short \& Stewart 1998). Past work (e.g. see 
(a)

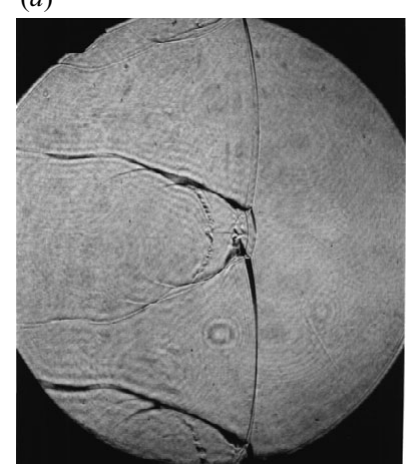

(b)

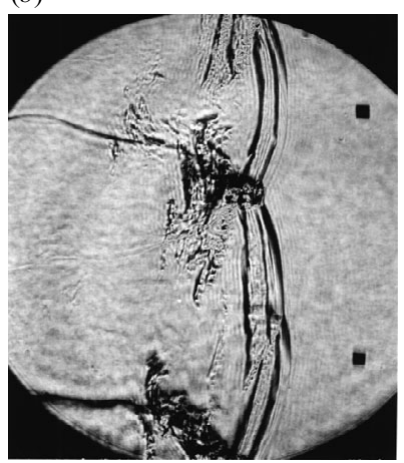

(c)

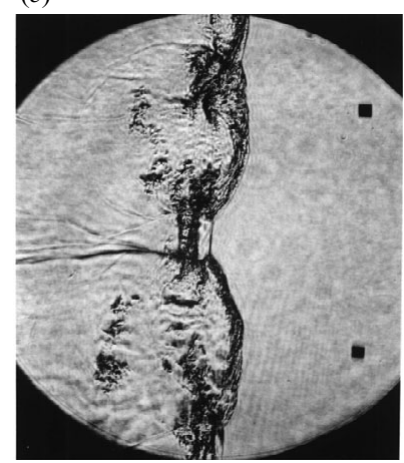

FiguRE 1. Pulsed laser schlieren images of detonation front propagating in $(a) 2 \mathrm{H}_{2}-\mathrm{O}_{2}-17 \mathrm{Ar}$, (b) $2 \mathrm{H}_{2}-\mathrm{O}_{2}-5.6 \mathrm{~N}_{2}$, (c) $\mathrm{C}_{3} \mathrm{H}_{3}-5 \mathrm{O}_{2}-9 \mathrm{~N}_{2}$, all at an initial pressure of $20 \mathrm{kPa}$. Detonations propagated left to right in a $152 \times 18 \mathrm{~mm}^{2}$, cross-section channel (consult Austin 2003; Austin et al. $2005 a$, for more details on the experiments).

discussions White 1961; Voitsekhovskii, Mitrofanov \& Topchian 1963; Edwards, Hooper \& Meddins 1972; Strehlow \& Crooker 1974; Takai, Yoneda \& Hikita 1974, Pintgen et al. 2003b) has revealed an extremely complex structure: the front is unstable in three-dimensions and also highly unsteady. The strength of the lead shock oscillates periodically in time and in space and detonations exhibit systems of counter-propagating transverse shock waves (figure 1). Triple points are formed at the intersection of the lead shock and transverse wave system. The triple-point shear layer separates premixed gas streams that have passed through portions of the lead shock with different strengths.

Regions of intense chemiluminescence (e.g. figure $2 a$ ) have recently been observed experimentally in detonation fronts propagating in highly unstable mixtures with high effective activation energy (Austin 2003; Austin et al. 2005b). From corresponding schlieren images, it appears the 'hotspots' occur in the vicinity of the triple-point shear layers in the latter portion of the detonation cell, prior to the triple point collision (Austin 2003). Such local hotspots were not observed in modest effective activation energy mixtures. Time-resolved imaging techniques have also revealed blast waves associated with localized explosions in high activation energy mixtures (Vasiliev \& Nikolaev 1978; Austin et al. 2005a). These localized explosions, the centre of which appears to occur some distance inside the previous cell cycle when a new cell is generated, may result from or be augmented by hotspot formation in the triple-point shear layers.

The magnitude of the lead shock oscillation through the cell cycle has been found to increase with increasing activation energy (Gamezo, Desbordes \& Oran 1999b; Austin et al. 2005a), while the transverse wave strength is calculated to remain relatively constant (Strehlow 1969; Urtiew 1970; Austin 2003). In high activation energy mixtures, a significant difference in the lead shock strength might therefore be expected at the triple point, resulting in a considerable disparity in the induction time across the shear layer. Any local decoupling of the detonation front near the end of the cell cycle increases the induction time difference even more significantly (Oppenheim, Smolen \& Zajac 1968; Pintgen, Austin \& Shepherd 2003a; Austin et al. $2005 a$ ). Thus in high activation energy mixtures, there is an increased probability that shear-layer instability may lead to formation of vortical structures before ignition 
(a)

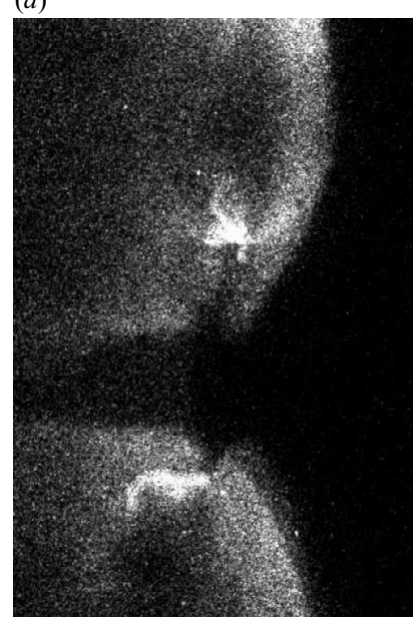

(b)

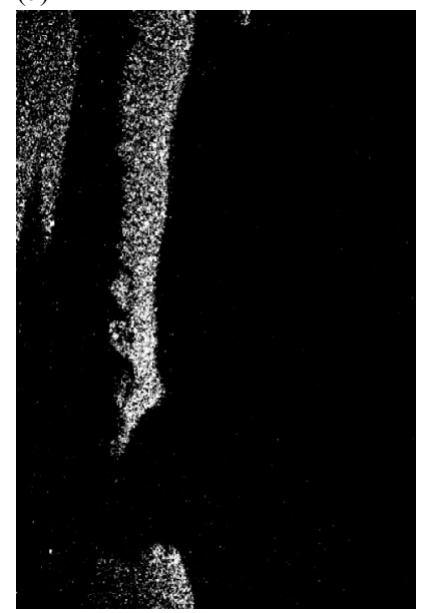

(c) Shear layer

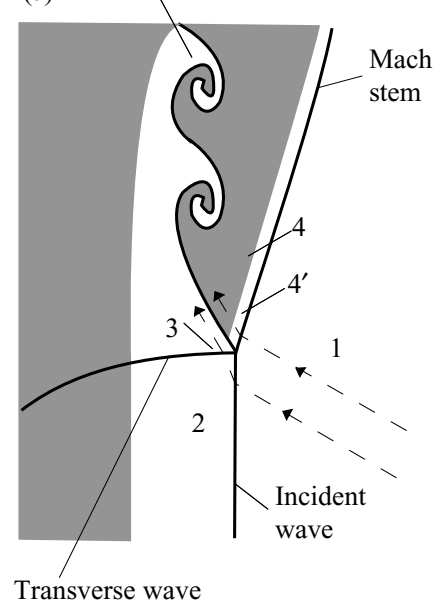

FiguRE 2. (a) Chemiluminescence images showing regions of high fluorescence intensity or 'hotspots' in a propagating gaseous detonation wave in a highly effective activation energy mixture, $\mathrm{C}_{3} \mathrm{H}_{8}-5 \mathrm{O}_{2}-9 \mathrm{~N}_{2}, P_{1}=20 \mathrm{kPa}$, (more details about the experiments can be found in Austin 2003; Austin et al. 2005a). Image height is $65 \mathrm{~mm}$. Simultaneous schlieren images indicate the hotspots occur in the vicinity of triple-point shear layers near the end of the cell cycle, but prior to the triple-point collision (see Austin 2003). (b) OH Planar laser-induced fluorescence image of a portion of a detonation front propagating in $2 \mathrm{H}_{2}-\mathrm{O}_{2}-7.7 \mathrm{~N}_{2}, P_{1}=$ $20 \mathrm{kPa}$, (Austin 2003). Kelvin-Helmholtz instabilities are evident between gases of different $\mathrm{OH}$ number density. The height of the frame is $30 \mathrm{~mm}$. (c) Schematic of a triple-point configuration with incident wave velocity $0.9 U_{C J}$ and inflow angle $33^{\circ}$, corresponding to conditions near the end of the cell cycle (Pintgen et al. 2003b). Dashed lines are particle paths. Grey shading indicates reacted gas. The unreacted gas in states 2 and 3 forms a 'keystone'-shaped reaction zone structure. The triple-point shear layer separates gas which has passed through the incident and transverse waves (state 3), and gas which has passed through the Mach stem (state $4^{\prime}$ ). For the simulations, gas at state $4^{\prime}$ is assumed to undergo a constant pressure reaction to state 4 .

occurs. Experiments by Austin (2003) and Pintgen et al. (2003b) have revealed a Kelvin-Helmholtz type instability between gas streams with different induction times in detonation waves, a phenomenon illustrated in figure $2(b)$. It is possible that transport of hot products and cold reactants across the shear layer may lead to hotspot formation and local explosions, critically augmenting the fundamental shockinduced combustion mechanism in the detonation front.

Motivated by these experiments, we examine the nature of the triple-point shear layer in a gaseous detonation wave. The shear layer consists of two premixed gas streams: one supersonic, unreacted stream that has passed through the incident and transverse waves, and one subsonic, reacted stream that has passed through the Mach stem (figure $2 c$ ).

The inflow conditions for the triple-point shear layer may be estimated from shock polar calculations once the triple-point structure is known. Detailed observations of cellular structure in marginal detonations were made by Gamezo et al. (2000). Numerical simulations in a high activation energy mixture examined a single transverse wave structure through the cell cycle and found that a transverse detonation was formed upon wall reflection of an unreactive transverse wave when the induction zone was large enough. In the case of a reactive transverse wave, a thin 'tail' of unreacted gas was observed near the secondary triple-point shear layer. When 
the transverse wave is unreactive, Kelvin-Helmholtz shear-layer instabilities were observed in the primary triple-point shear layer. Subbotin (1975) observed both reactive and unreactive transverse waves in high and low activation energy mixtures at different initial pressures. Time-resolved chemiluminescence images were combined with shock polar calculations to characterize the transverse wave structure. In the case of unreactive transverse waves, the shear layer separated reacted and unreacted gas forming a keystone-shaped region, whereas for detonative transverse waves this keystone region was significantly smaller and similar in appearance to the 'tails' observed by Gamezo et al. (2000). In the present experiments, soot foils for the same mixtures in the same channel show no evidence of detonative transverse waves (Austin 2003). A distinct keystone region is seen in figure 2(a), indicating that at least the upstream portion of the transverse wave is unreactive. The change in luminescence intensity across the transverse waves in the region downstream of the incident wave induction zone in figure 2(a) might lead as to suspect detonative waves; however, the corresponding schlieren image shows no cellular instability in this region. Thus for this work, we consider the case of a single triple point and unreactive transverse wave as the inflow condition for the shear layer. Vortical structures appearing in thin tails, as in the case of detonative transverse waves, are left for future study.

The shear-layer system is characterized by having two possible ignition fronts, one perpendicular to the flow and associated with the thermal runaway of the shocked reactants, the other parallel to the streams and driven by molecular heating across the thermal layer. In the latter case, the physics underlying the propagation of the combustion front is dominated by diffusion effects, and is of particular interest since diffusive processes are commonly thought to occur on time scales that are too long to be of relevance to detonations. Moreover, understanding the interaction between the front and the Kelvin-Helmholtz instability is fundamental to determining the influence of the instability on the explosion events.

The possible role of diffusion in detonation waves has been previously addressed in several papers. Gamezo, Desbordes \& Oran (1999a) estimated the importance of diffusion in consuming unreacted gas pockets some distance downstream of the lead shock. Singh, Leiberman \& Shepherd (2003) showed that molecular and thermal transport effects are negligible in one-dimensional detonation waves. Arienti \& Shepherd (2005) extended this work to evaluate the role of diffusion in triple-point shear layers in one-dimensional model problems. Time-resolved species evolution calculations using detailed chemical kinetics and simplified mixing models showed that the effect of diffusion depends on the mixture activation energy. Diffusion was negligible for modest activation energy mixtures, but decreased the induction time in high activation energy mixtures. A temperature bulge followed by a laminar diffusion flame appeared in high activation energy mixtures when thermal and mass diffusion was included in the numerical calculation.

All the works mentioned in the previous paragraph neglect the spatially developing structures associated with the shear-layer instability. Thus, the present investigation builds on existing work, but focuses on the relation between mixture chemistry and the shear-layer instability, with the objective of exploring the link between 'hotspot' formation and the effective activation energy. Three mixtures with markedly different effective activation energies are chosen to match experimental conditions (Austin 2003). Localized explosions were observed for the high-activation-energy case and, sporadically, for the medium, whereas uniform ignition was observed for the loweractivation-energy case. Since the induction distances are short, the linear stability characteristics of the shear layer are considered for the three mixtures. The results of 
the linear analysis are elaborated on by performing two-dimensional Navier-Stokes simulations with detailed chemistry and transport.

This paper is organized as follows. In $\S 2$, we present the initial conditions for the two gas streams in the three mixtures considered in this study. The chemistry model, effective activation energy of the mixtures considered, and the nature and time scales of transversal ignition are discussed in $\S 3$. A spatial linear stability analysis is carried out $(\S 4)$, including an examination of the effects of finite chemistry. A nonlinear analysis through numerical simulations of the two-dimensional Navier-Stokes equations with a detailed chemical kinetics mechanism is then presented in $\S 5$.

\section{Initial conditions: triple-point calculation}

The initial conditions for the two streams on either side of the shear layer are calculated using nonreactive shock polars. Shock polar calculations have been successfully used to analyse detonation triple points by, for example, Oppenheim et al. (1968); Urtiew (1970); Subbotin (1975); Pintgen et al. (2003b). The flow is assumed to be steady in the triple-point frame. Two parameters must be specified to close the calculation for conditions on either side of the contact surface. For all mixtures, the incoming velocity is taken to be $0.9 U_{C J}$, where $U_{C J}$ is the Chapman-Jouguet velocity. Experiments discussed in Voitsekhovskii et al. (1963), Lundstrom \& Oppenheim (1969), and Austin et al. (2005a) have demonstrated that $0.9 U_{C J}$ is representative of the incident wave velocity near the end of the cell cycle, the region of interest for this study. The incoming flow angle is taken to be $33^{\circ}$, a reasonable value based on soot foil track angles. More details on the triple-point calculation procedure can be found in Austin (2003).

Calculations show the triple-point shear layer separates a supersonic, relatively cold, gas stream and a subsonic, relatively hot, gas stream, states 3 and $4^{\prime}$, respectively, in figure $2(c)$. State 3 is found using chemically frozen shock calculations. In order to examine the role of mixing between unreacted and reacted gas streams as a potential mechanism for hotspot formation, and given the disparity in induction time between the hot (state $4^{\prime}$ ) and the cold (state 3) streams, we allow the subsonic stream to undergo a constant pressure reaction from the chemically frozen post-shock state (state $4^{\prime}$ ) to state 4 . This results in the initial conditions given in table 1 . The induction times for the two unreacted streams and the mixture effective activation energy based on state 3 are also reported in table 1. Three mixture with different effective activation energy were considered. The mixtures were chosen to correspond to experimental conditions of Austin (2003). In this paper, we will denote the mixture $2 \mathrm{H}_{2}-\mathrm{O}_{2}-17 \mathrm{Ar}$ as the hydrogen-oxygen-argon system, the $2 \mathrm{H}_{2}-\mathrm{O}_{2}-5.6 \mathrm{~N}_{2}$ mixture as the hydrogen-oxygen-nitrogen system, and the $\mathrm{C}_{3} \mathrm{H}_{8}-5 \mathrm{O}_{2}-9 \mathrm{~N}_{2}$ mixture as the propane system. The initial pressure for all mixtures was $20 \mathrm{kPa}$.

\section{Chemistry}

\subsection{Chemistry model}

Detailed chemical kinetics models deal with a large number of single-step reversible reactions; each reaction is characterized by an Arrhenius law correlating its rate of progress to the local thermodynamic state. Each rate expression is an optimized curve fit of a set of experimental data. In a computational analysis, the choice of the chemistry model is based on a trade-off between computational efficiency and accuracy. The chemistry model used in this study is the most recent release 


\begin{tabular}{ccccccc}
\hline $\begin{array}{c}\text { Mixture } \\
\text { initial }\end{array}$ & $\begin{array}{c}\rho_{3} \\
\left(\mathrm{~kg} \mathrm{~m}^{-3}\right)\end{array}$ & $\begin{array}{c}\rho_{4} \\
\left(\mathrm{~kg} \mathrm{~m}^{-3}\right)\end{array}$ & $\begin{array}{c}\boldsymbol{u}_{3} \\
\left(\mathrm{~m} \mathrm{~s}^{-1}\right)\end{array}$ & $\begin{array}{c}\boldsymbol{u}_{4} \\
\left(\mathrm{~m} \mathrm{~s}^{-1}\right)\end{array}$ & $T_{3}(\mathrm{~K})$ & $T_{4}(\mathrm{~K})$ \\
$2 \mathrm{H}_{2}-\mathrm{O}_{2}-17 \mathrm{Ar}$ & 1.23 & 0.780 & 1107 & 568.4 & 1682 & 2761 \\
$2 \mathrm{H}_{2}-\mathrm{O}_{2}-5.6 \mathrm{~N}_{2}$ & 1.12 & 0.577 & 1161 & 506.6 & 1302 & 2828 \\
$\mathrm{C}_{3} \mathrm{H}_{8}-5 \mathrm{O}_{2}-9 \mathrm{~N}_{2}$ & 2.08 & 0.867 & 1030 & 553.7 & 1522 & 3276 \\
& $\boldsymbol{a}_{3}$ & $\begin{array}{c}\theta_{\text {eff }} \\
\left(\mathrm{m} \mathrm{s}^{-1}\right)\end{array}$ & $-\left(\frac{\mathrm{d} \ln \tau_{i}}{\mathrm{~d} \ln T}\right)_{v N}$ & $\begin{array}{c}M_{c} \\
\text { (see Jackson \& } \\
\text { Grosch 1990) }\end{array}$ & $\begin{array}{c}\tau_{i, 3} \\
(\mu \mathrm{s})\end{array}$ & $\begin{array}{c}\tau_{i, 4^{\prime}} \\
(\mu \mathrm{s})\end{array}$ \\
$2 \mathrm{H}_{2}-\mathrm{O}_{2}-17 \mathrm{Ar}$ & 784.4 & 5.4 & 0.307 & 5.3 & 3.0 & $\begin{array}{c}\text { hydrogen-oxygen- } \\
\text { argon }\end{array}$ \\
$2 \mathrm{H}_{2}-\mathrm{O}_{2}-5.6 \mathrm{~N}_{2}$ & 800.3 & 6.9 & 0.346 & 7.8 & 2.6 & $\begin{array}{c}\text { hydrogen-oxygen- } \\
\text { nitrogen propane }\end{array}$ \\
$\mathrm{C}_{3} \mathrm{H}_{8}-5 \mathrm{O}_{2}-9 \mathrm{~N}_{2}$ & 711.2 & 12.7 & 0.256 & 10.2 & 1.4 &
\end{tabular}

TABLE 1. Calculated properties at the triple-point contact surface for the three mixtures considered in this study. State 3 is behind the incident and transverse waves and state 4 is after reaction from state $4^{\prime}$ behind the Mach stem (schematic is shown in figure $2 c$ ). The triple-point configuration is calculated using unreactive shock polars, as discussed in the text. $\theta_{\text {eff }}$ is the effective activation energy of the mixture, based upon change in the induction time, $\tau_{i}$, in response to a change in the lead shock velocity. $\theta_{\text {eff }}$ is calculated using the Konnov kinetics mechanism (Konnov 2000) as discussed in Schultz \& Shepherd (2000). $M_{c}$ is the convective Mach number. Velocities $u_{3}, u_{4}$ and the speed of sound, $a_{3}$, are in the triple-point frame. The entries in the lower-right corner of the table indicate the names used to refer to the three detonation systems in the rest of the paper.

of the GRI model, version 3.0, Smith et al. (2000), which includes 325 elementary chemical reactions and 53 species. In this version, propane and $\mathrm{C} 2$ oxidation products have been added. The accuracy of the mechanism in predicting detonation wave characteristics is analysed in this section by means of a comparison with another combustion mechanism for small hydrocarbon combustion, the Konnov mechanism (Konnov 2000). The Konnov mechanism includes 127 species and 1207 reactions, and has been previously validated for detonation temperatures and pressures by comparing the calculated ignition delay time with available shock tube data (Schultz \& Shepherd 2000).

The GRI mechanism is validated by comparison with the Konnov mechanism through the calculation of a Zel'dovich-von Neumann-Döring (ZND) detonation structure for the hot unreacted stream, state $4^{\prime}$, of the propane system shear layer. The results of the comparison are shown in fiugre 3. The GRI and Konnov mechanisms predictions for thermo-fluid variables and species mass fractions are in good agreement. Given the smaller size of the GRI 3.0 mechanism, it has been chosen for the simulations performed for this work.

\subsection{Transversal ignition}

In the experiments, the 'hotspot' event occurs in mixtures with large mixture effective activation energy. The effective activation energy is a global variable defined by the responsive change in the induction time to small changes in the post-shock conditions. The mixtures (table 1) are characterized on the basis of their effective activation energy, and are denoted as low, the hydrogen-oxygen-argon system, medium, the hydrogenoxygen-nitrogen system, and high, the propane system, activation energy mixtures. Higher activation energy mixtures exhibit increased lead shock oscillation through a 

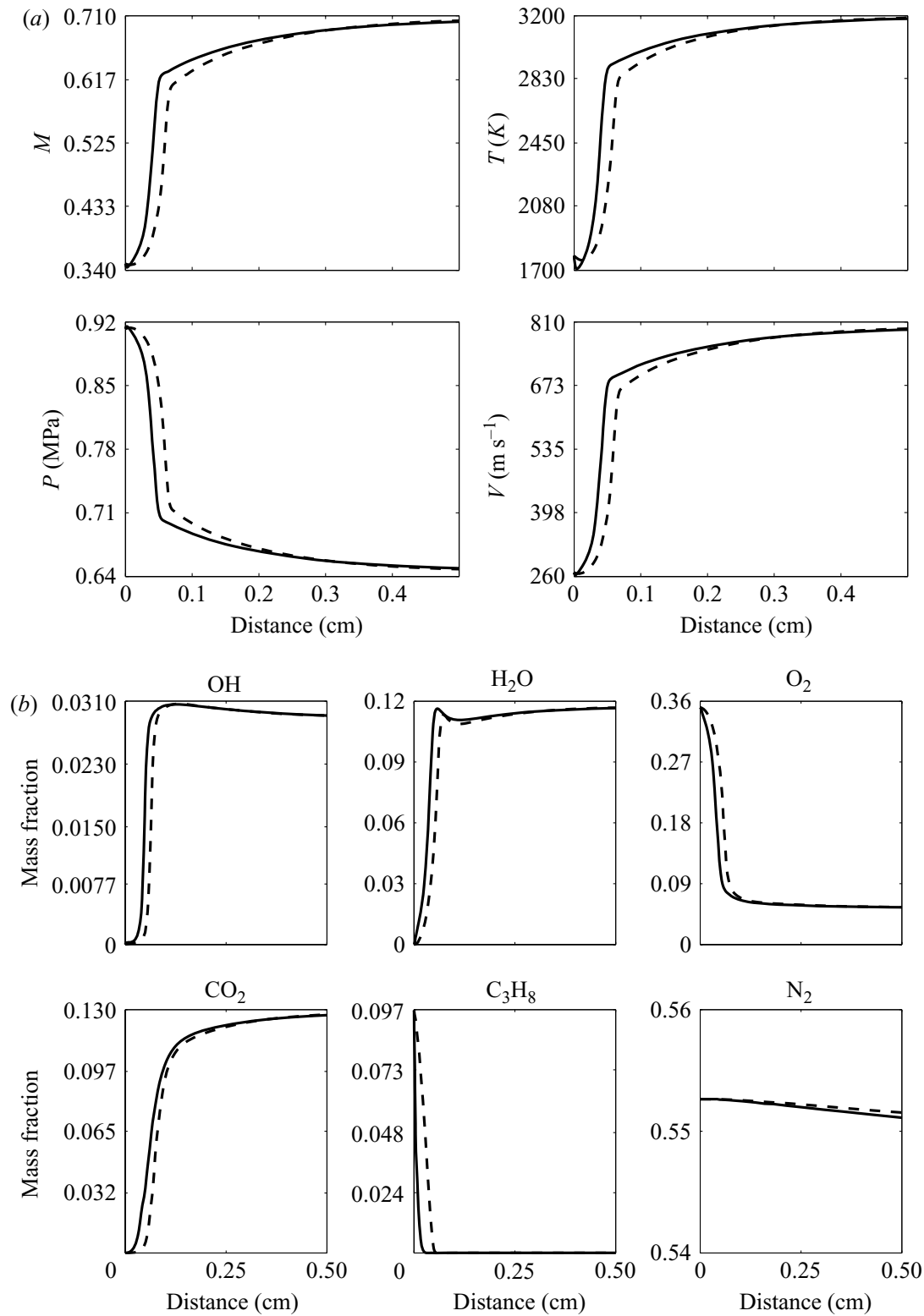

FIGURE 3. A comparison Konnov chemistry model (solid line) and the GRI chemistry model (dashed line). Prediction of the ZND detonation profile for the hot stream of $\mathrm{C}_{3} \mathrm{H}_{8}-5 \mathrm{O}_{2}-9 \mathrm{~N}_{2}$, initial pressure, $\mathrm{P}_{1}, 20 \mathrm{kPa}$. (a) Fluid dynamic variables. (b) Selected species mass fraction.

cell cycle resulting in increased induction time disparity across the shear layer. The relation between effective activation energy and constant volume induction times, $\tau_{i, 3}$ in table 1, shows that auto-ignition of the cold stream occurs at larger streamwise locations for mixtures with higher effective activation energy. Fluid close to the boundary between the hot and cold streams in the shear layer ignites earlier than the induction time, because of the effect of heat and molecular mass transfer across the interface. We label the ignition that propagates perpendicularly to the inflow velocity as transversal ignition. Because both streams are premixed and have identical chemical 

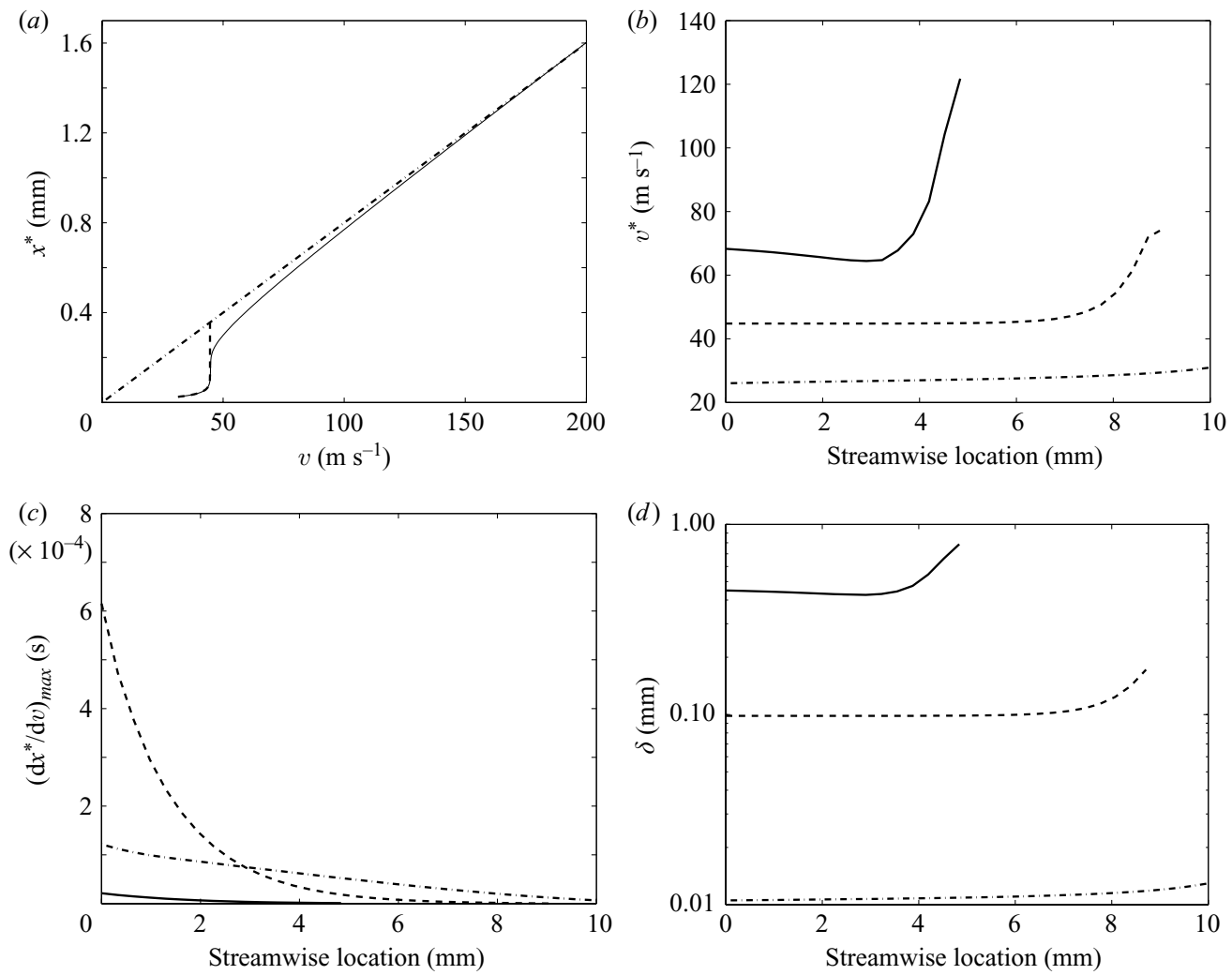

FIGURE 4. Transversal ignition calculated using a constant pressure one-dimensional deflagration analysis. (a) Ignition distance versus velocity. (b) Characteristic burning speed. (c) Derivative at the inflection point. (d) Flame thickness. (a) The mixture is the hydrogen-oxygennitrogen, with state 3 cold inflow conditions. $(b-d)$ lines: solid, hydrogen-oxygen-argon; dashed, hydrogen-oxygen-nitrogen; dash-dotted, propane system. Results are shown as a function of the streamwise location up to the end of the induction zone.

composition, a proper velocity scale for the transversal ignition process can be estimated as the adiabatic flame speed for a constant pressure deflagration supported by local conditions. The analysis is based upon the solution of the chemically reactive Navier-Stokes equations in quasi-one-dimensional form and for constant pressure (Buckmaster \& Ludford 1982). The results are summarized in figure 4, which is discussed in more detail in what follows. The principal difficulty in evaluating the adiabatic flame speed stems from the high reactivity of the cold stream, which causes the flame position $\left(x^{*}\right) v s$. velocity $(v)$ correlation to have an inflection point (solid line in figure $4 a$ ). This feature is explained by noticing that for large $v$ values, the solution is asymptotic to a straight line with slope $\tau_{i, 3} \dagger$ (dash-dotted line) while for low $v$ values, the solution is not affected by switching off the reaction term at temperatures close to the cold inflow temperature (dashed line in figure $4 a$ ). The proximity between the adiabatic flame speed evaluated using the switch-off reaction (dashed line) and the inflection point leads us to define a characteristic velocity as the location of the inflection point, $v^{*}$, corresponding to $v$ evaluated at $\max \left(\partial x^{*} / \partial v\right)$ of the solid line in (figure $4 a$ ).

$\dagger$ The constant pressure and constant volume induction times differ by less than $5 \%$, therefore, for brevity of notation, the latter is referenced in this section. 
Because of the assumption of adiabatic conditions, the burning velocity can be considered a local property. Therefore an obvious issue is to examine how $v^{*}$ varies with the streamwise coordinate. To this end we assume that the cold stream follows a constant pressure non-diffusive solution supported by the inflow conditions, state 3 . Such, solution is used to correlate $v^{*}$ with the streamwise coordinate. The results of this analysis are summarized in figures $4(b)$ to $4(d)$. Figure $4(b)$ shows the variation of $v^{*}$ with the streamwise coordinate for the three mixtures, figure 4(c) shows the corresponding variation of $\max \left(\partial x^{*} / \partial v\right)$, whereas figure $4(d)$ shows the flame thickness defined as

$$
\delta=\frac{T_{\max }-T_{\min }}{T_{\max }^{\prime}},
$$

where $T^{\prime}$ is the temperature derivative with respect to the spatial coordinate in the quasi-one-dimensional analysis.

The main results of the analysis are summarized below.

(i) The burning velocity decreases with increasing effective activation energy.

(ii) The propagation velocity of the transversal ignition front is significantly lower than the flow velocity. It is therefore unlikely that hotspot formation can be explained by simply considering lateral ignition of the unreacted stream. In fact, based on the average value of $v^{*}$ in figure $4(b)$, the distance the transversal front travels before reaching ignition is $0.3905 \mathrm{~mm}, 0.3761 \mathrm{~mm}$ and $0.2815 \mathrm{~mm}$ for the hydrogen-oxygenargon, hydrogen-oxygen-nitrogen, and propane systems, respectively. A comparison between the length scales obtained in this section and those based on a twodimensional analysis is given in $\S \S 4.6$ and 5.2.2;

(iii) The lower the activation energy, the faster $\max \left(\partial x^{*} / \partial v\right)$ approaches the induction time, $\tau_{i}$, cf figure 4(c). In order for local conditions at the shear-layer interface to support an adiabatic propagating front, the ratio between $\max \left(\partial x^{*} / \partial v\right)$ and $\tau_{i}$ must be large. The results of figure 4(c) suggest that transversal ignition plays a lesser role for low activation energies and large streamwise distances, i.e. in such conditions, the mixture is more likely to react as a convective explosion.

\section{Linear stability analysis}

The objective of the analysis presented in this section is to investigate the nature of the Kelvin-Helmholtz instability in different mixtures and to isolate the effect of the activation energy on the associated growth rate. The merit of the linear analysis in describing mixing-layer instability has been shown in recent years by comparing full Navier-Stokes and linear perturbation results to experimental measurements, see for example, Criminale, Jackson \& Joslin (2003). The linear analysis is relevant to this study because, owing to the short induction times, any interaction between mixing and gas ignition will occur close to the triple point. In order for vortical mixing to occur between the cold unreacted stream and the hot reacted stream, the instability must develop upstream of the induction distance for the cold stream. Therefore, the analysis is restricted to the region preceding auto-ignition in the unreacted stream, and the emphasis is on the relation between the chemistry and the eigenvalue growth rate in the pre-explosion region.

A linear stability analysis of a reacting mixing layer was performed in Shin \& Ferziger (1993) for a constant caloric properties system with simplified one-step kinetics. The approach adopted in the present work is to preserve the caloric and chemical complexity of the system, thus offering a realistic insight into the development of the instabilities. 


\subsection{Mean flow}

The linear analysis centres around the partition of the inherently unsteady flow into a stationary mean and a perturbation part. By virtue of the restriction of the analysis to the region before auto-ignition, chemistry affects the mean flow by influencing transversal ignition. The mean flow is defined by the two-dimensional compressible Navier-Stokes equation in the boundary-layer form which neglects pressure gradients, retains only the mixed $\sigma_{x y}$ component of the shear stress, and considers only the $y$ component of the heat flux and species diffusive fluxes. In what follows, $N_{s}$ represents the number of species in the model and $N_{e l}$ is the number of atomic elements. In dimensional form, the equations are,

$$
\begin{gathered}
\frac{\partial \rho u}{\partial x}+\frac{\partial \rho v}{\partial y}=0, \\
\rho u \frac{\partial u}{\partial x}+\rho v \frac{\partial u}{\partial y}=\frac{\partial}{\partial y}\left(\mu \frac{\partial u}{\partial y}\right), \\
\rho u \frac{\partial h}{\partial x}+\rho v \frac{\partial h}{\partial y}=\frac{\partial}{\partial y}\left(\lambda \frac{\partial T}{\partial y}\right)+\sum_{k=1}^{N_{s}} \frac{\partial}{\partial y}\left(\sum_{j=1}^{N_{s}}\left(v_{j, k} h_{j}\right) \frac{\partial Y_{k}}{\partial y}\right)+\mu\left(\frac{\partial u}{\partial y}\right)^{2}, \\
\rho u \frac{\partial Y_{j}}{\partial x}+\rho v \frac{\partial Y_{j}}{\partial y}=\sum_{k=1}^{N_{s}} \frac{\partial}{\partial y}\left(v_{j, k} \frac{\partial Y_{k}}{\partial y}\right)+\rho \Omega_{j} \quad\left(j=1, \ldots, N_{s}\right) .
\end{gathered}
$$

This set of equations is completed by the thermal and caloric equations of state, the imposition of the constant pressure and the rate term Arrhenius approximations, $\dot{\omega}_{j}$,

$$
\begin{gathered}
P=P_{o}, \\
T=T\left(P, h, Y_{j}\right), \\
\rho=P /\left(T \sum_{k=1}^{N_{s}} R_{k} Y_{k}\right), \\
\Omega_{j} \equiv \dot{\omega}_{j} / \rho=\Omega_{j}\left(P, h, Y_{k}\right) \quad\left(j=1, \ldots, N_{s}\right) .
\end{gathered}
$$

A solution of the mean flow equation is carried out by first introducing the HowarthDorodnitzyn variables,

$$
\begin{gathered}
\tilde{y}=\int_{0}^{y} \rho \mathrm{d} t, \\
\tilde{v}=\rho v+u \int_{0}^{y} \rho_{x} \mathrm{~d} t,
\end{gathered}
$$

which transform (4.1) into

$$
\begin{gathered}
\frac{\partial u}{\partial x}+\frac{\partial \tilde{v}}{\partial \tilde{y}}=0 \\
u \frac{\partial u}{\partial x}+\tilde{v} \frac{\partial u}{\partial \tilde{y}}=\frac{\partial}{\partial \tilde{y}}\left(\rho \mu \frac{\partial u}{\partial \tilde{y}}\right) \\
u \frac{\partial h}{\partial x}+\tilde{v} \frac{\partial h}{\partial \tilde{y}}=\frac{\partial}{\partial \tilde{y}}\left(\rho \lambda \frac{\partial T}{\partial \tilde{y}}\right)+\sum_{k=1}^{N_{s}} \frac{\partial}{\partial \tilde{y}}\left(\rho \sum_{j=1}^{N_{s}}\left(v_{j, k} h_{j}\right) \frac{\partial Y_{k}}{\partial \tilde{y}}\right)+\mu \rho\left(\frac{\partial u}{\partial \tilde{y}}\right)^{2}, \\
u \frac{\partial Y_{j}}{\partial x}+\tilde{v} \frac{\partial Y_{j}}{\partial \tilde{y}}=\sum_{k=1}^{N_{s}} \frac{\partial}{\partial \tilde{y}}\left(\rho v_{j, k} \frac{\partial Y_{k}}{\partial \tilde{y}}\right)+\Omega_{j} \quad\left(j=1, \ldots, N_{s}\right) .
\end{gathered}
$$


Here, $v_{i, k}$ are the effective multicomponent diffusion coefficients. The word 'effective' is used to indicate that the coefficients directly multiply the mass fraction gradients. $v_{i, k}$ are related to the ordinary multicomponent diffusion coefficients, $\tilde{v}_{j, l}$, by

$$
v_{j, l}=\rho m_{j}\left(\sum_{k=1}^{N_{s}} \frac{\tilde{v}_{j, k}}{m_{l}}-\frac{\tilde{v}_{j, l}}{\hat{m}}\right) \quad\left(j=1, \ldots, N_{s}, l=1, \ldots, N_{s}\right),
$$

with $m_{j}$ and $\hat{m}$ the species and mixture mean molecular weights, respectively. The ordinary diffusion coefficients are obtained from the binary diffusion coefficients using the 'extended' Chapman-Enskog procedure detailed in Dixon-Lewis (1968). Likewise, the other transport coefficients, which include the mixture average thermal conductivity, $\lambda$, are based on kinetic theory approximate solutions and are determined using a CHEMKIN based multicomponent transport package (Kee et al. 1986, 1996).

In this form, the only difference between the system composed by the momentum and continuity equation and its incompressible non-reactive analogue is the dependence of the product $\rho \mu$ on the species mass fractions and temperature. To simplify the linear stability analysis, we assume that the product is constant and equal to its free-stream value,

$$
\rho \mu=(\rho \mu)_{\infty},
$$

(see Lock 1951). A comparison of the linear stability characteristics for a non-reactive compressible mixing layer using the Lock model $(\rho \mu=$ const $)$ and Sutherland's model $\left(\mu=a T^{3 / 2} /(b+T)\right.$ where $a, b$ are constants) was made by Jackson \& Grosch (1991). A key finding of this work is that the growth rates, when properly normalized, are essentially independent of the choice of the model for the viscosity $\mu$. Since the Lock model is computationally faster, we chose this for the results reported here without loss of generality. This Lock model approximation leads to the solution of the velocity components in terms of the similarity variable,

$$
\eta=\sqrt{\left(\frac{u}{2 \rho \mu}\right)_{\infty}} \frac{\tilde{y}}{\sqrt{x}}
$$

by seeking a similarity solution for which the zero (originating at the triple point) streamline is horizontal,

The similarity equation is

$$
u=F^{\prime}(\eta) u_{\infty}
$$

$$
F^{\prime \prime \prime}+F F^{\prime \prime}=0
$$

with boundary conditions:

$$
\begin{gathered}
F^{\prime}(\infty)=1, \\
F(0)=0, \\
F^{\prime}(-\infty)=\beta_{u} \equiv u_{-\infty} / u_{\infty} .
\end{gathered}
$$

A solution depending only on the similarity variable $\eta$ is clearly not possible for the temperature and species, as is easily seen by taking the limit $\eta \rightarrow \infty$ of $(4.4 d)$ at the inflow plane. Because $\Omega_{i} \neq 0$, it follows that $\left(\partial Y_{i} / \partial x\right)_{\eta} \neq 0$; it is therefore the premixed nature of the combustion problem that prevents similarity solutions in $\eta$. Consequently, a similarity solution does not exist for the velocity vector itself, but only for the transformed velocity, (4.3), by virtue of the approximation in (4.6). This conclusion can be better understood by considering the effect of chemical reaction on the dilatation field, and thereby on the streamwise acceleration. 
The species and temperature equations are solved numerically by means of a pseudotime-marching algorithm, using the self-similar velocity field evaluated a priori at the mesh points. The computational mesh used for the mean flow computation is of size [200 $\times 500]$, and the solution domain is the box $\left\{(x, \eta) \mid x-x_{0} \in[0,0.5], \eta \in\right.$ $[-20,20]\}$. Here, $x_{0}$ is the distance between the inflow boundary and the detonation triple point; we take $x_{0}=0.1 \mathrm{~cm}$. For this distance, the term $\sqrt{(\operatorname{Rex} / L)} / 2$ takes values of 270 for the propane system, 175 for the hydrogen-oxygen-argon system, and 214 for the hydrogen-oxygen-nitrogen system. At the top and bottom boundaries of the domain, i.e. for $\eta= \pm 20$, the temperature and concentration $\eta$ derivatives approach zero, therefore proper boundary conditions are evaluated by solving for adiabatic explosion waves supported by the inflow. Inflow boundary conditions are determined by solving the non-reactive equation with constant caloric properties and unitary Prandtl and Schmidt numbers. (Note: unitary Prandtl and Schmidt numbers are only used to determine the inflow conditions, not the base flow.) Thermal and mass diffusivities are related to the viscosity by

$$
\frac{\rho \lambda}{c_{p}}=(\rho \mu)_{\infty}, \quad \rho v_{i, k}=(\rho \mu)_{\infty} \delta_{i, k},
$$

where $\delta$ is the Kronecker symbol. The following injection conditions are obtained:

$$
\begin{aligned}
T /(T)_{\infty} & =K_{1,0} F^{\prime}+K_{2,0}-\frac{(\gamma)_{\infty}-1}{2} M^{2}\left(F^{\prime}\right)^{2}, \\
Y_{i} & =K_{1, i} F^{\prime}+K_{2, i},
\end{aligned}
$$

where the constants $K_{j, i}$ are obtained by satisfying the conditions at $\eta= \pm \infty$. Zero gradient boundary conditions are used for the outflow boundary.

\subsection{Mean-flow results}

The mean-flow profiles are shown in figure 5 for a distance $x-x_{0}=0.25 \mathrm{~cm}$, approximately half of the smallest constant volume induction distance among the three systems, $\left(\tau_{i} u_{3}\right)_{\min }=0.58 \mathrm{~cm}$ corresponding to the low activation energy case. The solid curves represent the ratios $T / T_{\infty}$, while lines of other types represent selected species mass fractions that are normalized using the values on the hot side of the shear layer, $\eta=-\infty$. The size of the region where molecular diffusion is significant is of similar magnitude for the three mixtures and it extends over $\eta \in[-5,5]$. The location of the transversal ignition front is easily identifiable only for the high activation energy mixture, whereas for the other mixtures, the region of chemical activity is spread over the mixing region, a result expected based on the analysis at the end of $\S 3.2$.

A local maximum in temperature and $\mathrm{OH}$ mass fraction is observed at $\eta=0$ for the two lower activation energy cases. This outcome is partly due to the contribution of viscous heating, the last term on the right-hand side of (4.4c), which has a global maximum at $\eta=0$, corresponding to the maximum streamwise velocity derivative. The absence of the local maximum feature in the high activation energy case is related to the much larger ratio between heat release and viscous heating. The associated overshoot in the $\mathrm{OH}$ mass fraction is attributed to the temperature activated radical production. The absence of this feature in the propane system is a clear consequence of the much smaller flame thickness, cf. figure $4(d)$, so that, away from the ignition point, virtually all the reactant is consumed.

The interaction between transversal ignition and viscous heating for the two lower activation energy systems means that it is not possible to identify the location of the ignition front based upon the maximum rate of $\mathrm{OH}$ formation or the maximum 

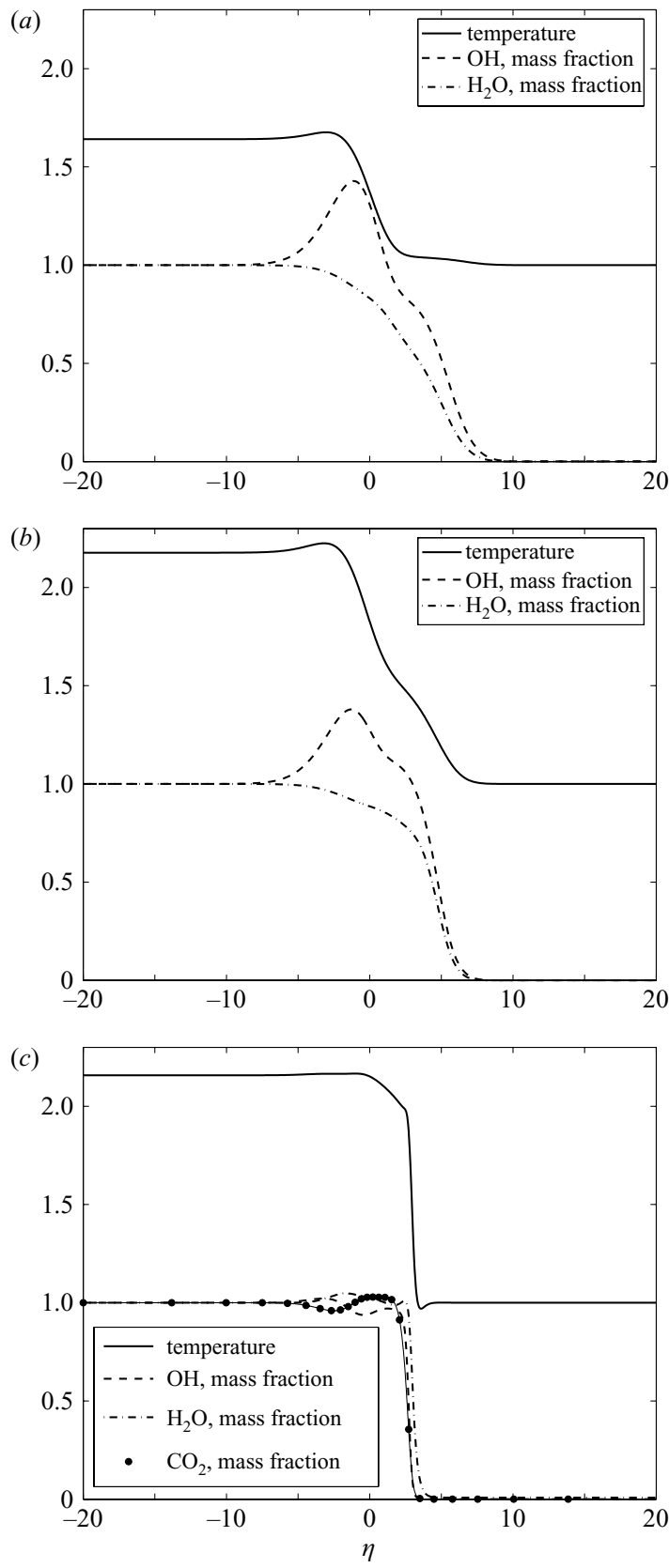

Figure 5. Mean flow at a location $x-x_{0}=0.25 \mathrm{~cm}$. The mass fractions are normalized by the values on the hot side of the layer $(\eta=-\infty)$, while temperature is normalized by $T_{\infty}$. (a) Hydrogen-oxygen-argon system. (b) Hydrogen-oxygen-nitrogen system. (c) Propane system.

temperature gradient. The bulge in $\mathrm{OH}$ mass fraction is stationary and increases in magnitude when the activation energy is decreased. The mean-flow results demonstrate a greater interaction between the chemistry and fluid mechanics for the low activation energy mixture, a consequence of the much larger transversal flame thickness. 
For the high activation energy system, the effect of the transversal front on the thermal and concentration fields leads to the identification of two regions in which solution gradients are significant: the region of high dynamic shear, associated with the maximum velocity gradient and located around $\eta=0$, and the region of high thermal and concentration diffusive flux associated with the transversal front. Unique among the three systems, the propane system shows a temperature undershoot on the cold side of the shear layer. This phenomenon, which is evident also in figure $3(a)$, is associated with the initial fuel decomposition reactions, and will be noted also in the nonlinear analysis.

\subsection{Inviscid perturbation}

The previously computed mean-flow profiles are used in a perturbation analysis by considering a parallel flow in chemical equilibrium and the perturbations to such equilibrium. We use the Euler equations to describe the perturbations since it is well known that inviscid disturbances dominate shear-layer instability (see Criminale et al. 2003). In the reminder of this section, spatial variations are non-dimensionalized using $L_{s}=\sqrt{x(2 \mu /(\rho u))_{\infty}}$ as the length scale. The velocity, pressure, density and temperature are non-dimensionalized by the corresponding values at $\eta=+\infty$, the enthalpy by the term $\left(c_{p} T\right)_{\infty}$, where $c_{p}$ is the M28 averaged specific heat. The species gas constants $R_{j}$, and the specific heats are non-dimensionalized by the mixture average specific heat at plus infinity, and the ratio between velocity scale and length scale, $(u)_{\infty} / L_{s}$, is used for the reaction rates $\Omega_{j}$. The following expansions are considered:

$$
\left[u, v, p, h, \rho, Y_{j}, \Omega_{j}\right]=\left[\bar{u}, 0,1, \bar{h}, \bar{\rho}, \bar{Y}_{j}, \bar{\Omega}_{j}\right]+\left[f, \alpha \phi, \Pi, \theta, r, z_{j}, w_{j}\right] \exp (\mathrm{i} \alpha(x-c t)),
$$

where the bar indicates a mean flow quantity, $\alpha$ is the spatial growth rate, and $c$ is the phase speed with frequency $\omega=\alpha c$. Here, we are interested in disturbances that grow in the downstream direction so $\alpha=\alpha_{r}+\mathrm{i} \alpha_{i}$ is complex with $\left(-\alpha_{i}\right)$ the growth rate of the spatial disturbance, and $\omega$ is real. Noting that the prime denotes differentiation with respect to the shear-layer variable $\eta$, the equations for the perturbations are written as:

$$
\begin{gathered}
\mathrm{i} \frac{(\bar{u}-c)}{\bar{\rho}} r+\mathrm{i} f+(\bar{\rho} \phi)^{\prime}=0, \\
\bar{\rho}\left[\mathrm{i}(\bar{u}-c) f+\bar{u}^{\prime} \bar{\rho} \phi\right]+\frac{\mathrm{i} \Pi}{\gamma_{\infty} M^{2}}=0, \\
\mathrm{i} \alpha^{2}(\bar{u}-c) \phi+\frac{\Pi^{\prime}}{\gamma_{\infty} M^{2}}=0, \\
\bar{\rho}\left[\mathrm{i}(\bar{u}-c) \theta+\bar{h}^{\prime} \bar{\rho} \phi\right]-\mathrm{i} \frac{\gamma_{\infty}-1}{\gamma_{\infty}}(\bar{u}-c) \Pi=0, \\
\mathrm{i} \alpha(\bar{u}-c) z_{j}+\bar{Y}_{j}^{\prime} \bar{\rho} \phi \alpha=w_{j} \quad\left(j=1, \ldots, N_{s}\right) .
\end{gathered}
$$

The perturbations are correlated through a set of algebraic relations dependent on the chemistry model,

$$
\begin{gathered}
r=\bar{\rho} \Pi+\bar{\rho}^{2} \frac{\gamma_{\infty}}{\gamma_{\infty}-1} \sum_{k=1}^{N_{s}}\left(\frac{R}{c_{p}} \bar{h}_{k}-R_{k} \bar{T}\right) z_{k}-\frac{\bar{\rho}}{c_{p} \bar{T}} \theta, \\
{\left[w_{1}, w_{2}, \ldots, w_{N_{s}-1}, w_{N_{s}}\right]^{T}=\Omega_{p} \Pi+\Omega_{h} \theta+\Omega_{y}\left[z_{1}, z_{2}, \ldots, z_{N_{s}-1}, z_{N_{s}}\right]^{T},}
\end{gathered}
$$


where

$$
\Omega_{p}=\left[\frac{\partial \bar{\Omega}_{1}}{\partial p}, \frac{\partial \bar{\Omega}_{2}}{\partial p}, \ldots, \frac{\partial \bar{\Omega}_{N_{s}-1}}{\partial p}, \frac{\partial \bar{\Omega}_{N_{s}}}{\partial p}\right]^{T}, \quad \Omega_{h}=\left[\frac{\partial \bar{\Omega}_{1}}{\partial h}, \frac{\partial \bar{\Omega}_{2}}{\partial h}, \ldots, \frac{\partial \bar{\Omega}_{N_{s}-1}}{\partial h}, \frac{\partial \bar{\Omega}_{N_{s}}}{\partial h}\right]^{T}
$$

are vectors of size $N_{s}$, while $\Omega_{y}$ of components $\partial \bar{\Omega}_{i} / \partial \bar{Y}_{j}$ is a Jacobian matrix of size $N_{s} \times N_{s}$. The source term vector $\bar{\Omega}$ and its derivatives, $\Omega_{p}, \Omega_{h}$ and $\Omega_{y}$ are evaluated using the GRI chemistry mechanism.

\subsection{Solution of the perturbation equation}

As in the case of a non-reactive mixture, the eigenvalue problem associated with the amplification of inviscid perturbations leads to a second-order homogeneous equation for the pressure perturbation,

$$
\Pi^{\prime \prime}+B \Pi^{\prime}+C \Pi=0
$$

The coefficients are described in terms of the matrix $\mathbf{S}$ of size $3 \times N_{s}$ with row entries:

$$
\begin{aligned}
S_{1,1: N_{s}} & =\left[\mathrm{i} \alpha(\bar{u}-c) \mathscr{I}-\Omega_{y}\right]^{-1}\left(-\alpha\left[\bar{Y}_{1}^{\prime}, \bar{Y}_{2}^{\prime}, \ldots, \bar{Y}_{N_{s}-1}^{\prime}, \bar{Y}_{N_{s}}^{\prime}\right]^{T}\right), \\
S_{2,1: N_{s}} & =\left[\mathrm{i} \alpha(\bar{u}-c) \mathscr{I}-\Omega_{y}\right]^{-1} \Omega_{p}, \\
S_{3,1: N_{s}} & =\left[\mathrm{i} \alpha(\bar{u}-c) \mathscr{I}-\Omega_{y}\right]^{-1} \Omega_{h},
\end{aligned}
$$

and the coefficients

$$
\begin{aligned}
s_{j} & =\rho^{2} \frac{\gamma_{\infty}}{\gamma_{\infty}-1} \sum_{k=1}^{N_{s}}\left(\frac{R}{c_{p}} \bar{h}_{k}-R_{k} T\right) S_{j, k}+t_{j} \quad(j=1,2,3), \\
\boldsymbol{t} & =\left[\begin{array}{c}
0 \\
\bar{\rho} \\
-\bar{\rho} /\left(c_{p} \bar{T}\right)
\end{array}\right],
\end{aligned}
$$

where $\mathscr{I}$ is the identity matrix. Thus,

$$
\begin{aligned}
& B=-\left[\frac{s_{3} \bar{h}^{\prime}-\bar{\rho}^{\prime}}{\bar{\rho}}+2 \frac{\bar{u}^{\prime}}{\bar{u}-c}-\mathrm{i} s_{1} \frac{\bar{u}-c}{\bar{\rho}}\right], \\
& C=-\frac{\alpha^{2}}{\bar{\rho}^{2}}\left[1-M^{2}(\bar{u}-c)^{2}\left(s_{2} \gamma_{\infty}+\frac{s_{3}}{\bar{\rho}}\left(\gamma_{\infty}-1\right)\right)\right] .
\end{aligned}
$$

Introducing the Riccati variable (see Criminale et al. 2003, p. 145),

$$
G \equiv \frac{\Pi^{\prime}}{\Pi \alpha \bar{T}}
$$

the second-order equation is reduced to a first-order nonlinear equation

$$
G^{\prime}+\alpha \bar{T} G^{2}+\left(\frac{\bar{T}^{\prime}}{\bar{T}}+B\right) G+\frac{C}{\alpha \bar{T}}=0
$$

The boundary conditions are obtained by analysing the asymptotic trend of $\Pi$ in the free stream. Noting that the coefficient B vanishes on either side of the free stream, appropriate boundary conditions for the pressure perturbation are (Jackson \& Grosch 1989),

$$
\eta \rightarrow \pm \infty, \quad \Pi \rightarrow K_{ \pm \infty} \exp (\mp \sqrt{-C \eta})
$$

Boundary conditions for $G$ on both sides of the free stream are obtained by using (4.27) and (4.25). 

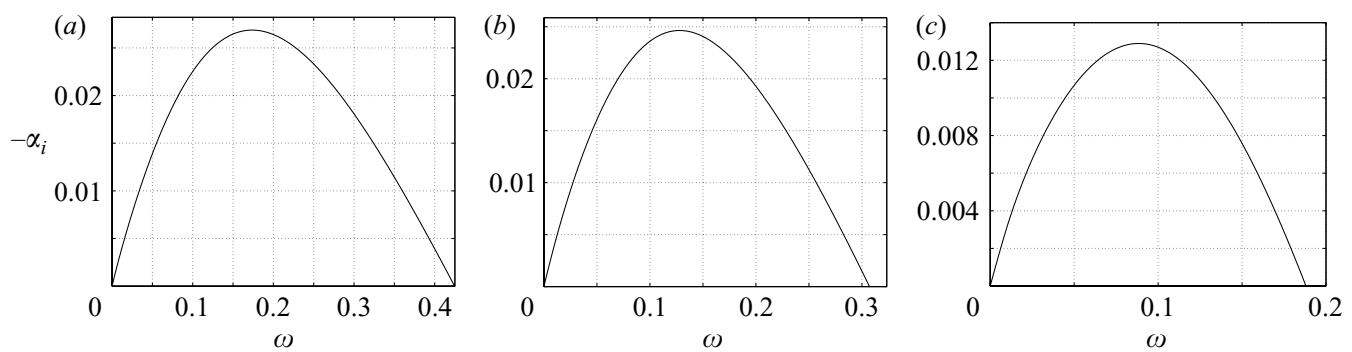

FIGURE 6. Spatial growth rate of inviscid perturbation to the mean flow for $(a)$ hydrogenoxygen-argon (b) hydrogen-oxygen-nitrogen and $(c)$ propane systems.

\subsubsection{Numerical algorithm}

The Riccati equation is integrated in the interval $\eta \in[-20,20]$, using a $(7,8)$ order variable step (adaptive) Runge-Kutta method, with an error tolerance of 1 $\times 10^{-10}$ (Shampine, Gladwell \& Brankin 1991). A sensitivity study to the size of the domain reveals that doubling the solution interval yields a maximum change of the 'normalized' pressure perturbation eigenfunction lower than $1 \times 10^{-9}$ for $\eta \in[-20,20]$ and for all the cases presented in the following sections. To represent the computed mean flow onto the grid points of the variable step Runge-Kutta, a quintic natural spline representation of the mean flow is used, so that locally in the interval between the knots $i$ and $i+1$ a mean flow variable $\bar{v}$ is related to $p \equiv \eta-\eta_{i}$ through the quintic polynomial:

$$
\bar{v}-\bar{v}_{i}=\left(\left(\left(\left(F_{i} p+E_{i}\right) p+D_{i}\right) p+C_{i}\right) p+B_{i}\right) p,
$$

where the coefficients $B_{i}, C_{i}, D_{i}, E_{i}, F_{i}$ are obtained enforcing continuity in the derivatives (up to the fourth order) at the knots. Both the mean flow state variables and their derivatives are interpolated using splines. The value of the mean flow derivatives are evaluated at the knots using fourth-order finite-difference formulae. With the grid considered in this study, the error associated with the spline representation of the mean flow was shown to be small when the algorithm was tested on a mean profile where the velocity is defined by a hyperbolic tangent relation and the temperature and species by (4.12). The disturbance equations are solved by integrating from $\eta=20$ to 0 , and integrating from $\eta=-20$ to 0 , then iterating on the eigenvalue until the pressure perturbation is continuous at $\eta=0$ using Muller's method, which is essentially a secant method for complex eigenvalues. A spatial stability study is pursued so that perturbation are allowed to grow in space but not in time. The eigenvalues are found for time frequencies $\omega=\alpha c$ on the real axis.

\subsection{Spatial growth rate}

Only one unstable eigenmode was found for each of the three mixtures considered. The non-dimensional growth rate, $-\alpha_{i}$ is plotted versus $\omega$ for the three mixtures in figure 6 . The set of figures shows that, in non-dimensional units, the disturbance growth rate decreases with an increase in effective activation energy. In dimensional units, $\left(-\alpha_{i}\right)_{\max }$ is equal to $14.2 \mathrm{~cm}^{-1}, 15.4 \mathrm{~cm}^{-1}$ and $9.8 \mathrm{~cm}^{-1}$ for the low, medium and large activation energy systems, respectively. The dimensional growth rate is, therefore, maximum for the medium activation energy case. This outcome is explained by considering the contrasting effects that an increase in the mixture activation energy has on the difference in injection flow conditions across the shear layer and on the perturbation dynamics. The former is linked to variations in the convective Mach number, velocity 

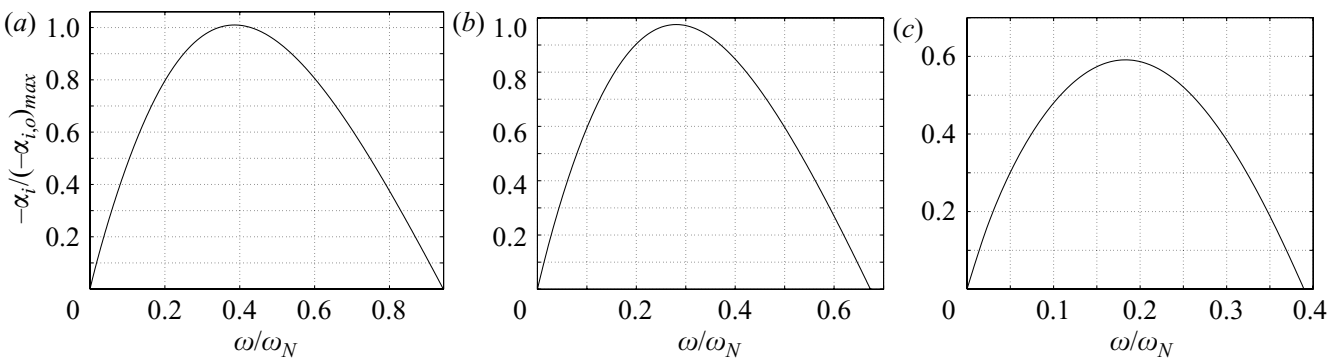

Figure 7. Comparison of the spatial stability eigenvalue with that of frozen systems having comparable fluid dynamic parameters. (a) Hydrogen-oxygen-argon (b) hydrogen-oxygennitrogen and $(c)$ propane systems.

and temperature ratios across the layer. It is discussed in and $\S 1$ documented in table 1. The latter effect, which appears to be dominant for large effective activation energies, is examined in detail in the remaining part of this section.

In order to isolate the effect of the mixture on perturbation dynamics, changes in shear-layer conditions are taken into account by comparing the growth rate to that of a dynamically similar, chemically frozen shear layer having as chemical composition that of the fresh mixture.

For this comparison, the frozen mean velocity is found using the well-known Blasius profile (Rosenhead 1963), whereas a self-similar profile is assumed for the mean temperature (equation (4.12)). The caloric and thermal properties of the mixture are kept constant and identical to their frozen state. Note that Jackson \& Grosch (1991) have shown that the use of a rheological equation rather than the Lock approximation, (4.6), has a negligible effect on the normalized growth rate. Similarly, based upon frozen flow eigenvalues computations, the effect of Prandtl-number differences among the three systems is assumed to be of modest importance for the range of values characteristic of the three mixtures. Consequently, the frozen flow stability is carried with $P r=1$ for all the mixtures.

Figure 7 shows the reactive system growth rate scaled by the corresponding maximum value for frozen chemistry, $\left(-\alpha_{i, 0}\right)_{\max }$, and plotted versus the ratio $\omega / \omega_{N}$, where $\omega_{N}$ represents the neutral frequency of the frozen modes. The results shown in figure 7 allow us to draw the following conclusion about the mixture effects on the linear spatial growth of disturbances in the premixed shear layer supported by a detonation wave. Chemistry and mixture effects have a strong effect in reducing the instability in reactive systems, both with regard to the maximum growth rate and the range of frequency of the unstable modes; this phenomenon, together with the correlation between induction time difference and effective activation energy, suggests that intermediate activation energy mixtures are more susceptible to the growth of perturbations in detonation shear layers.

The eigenfunctions are discussed in $\S 4.6$. The relation between chemical reaction time scales and mode spatial growth will be further elucidated in the following subsections by looking at the effect of finite-rate chemistry in the perturbation equation.

\subsection{Eigenfunctions}

The eigenfunctions for the most unstable modes, maximum value of $-\alpha_{i}$, are considered in this section. The eigenfunctions are normalized according to the condition, $\Pi(0)=1$. Figure 8 shows the eigenfunctions for the three mixtures in terms of $\Pi$ and $\alpha \phi$. An increase in the mixture activation energy leads to noticeable changes in 

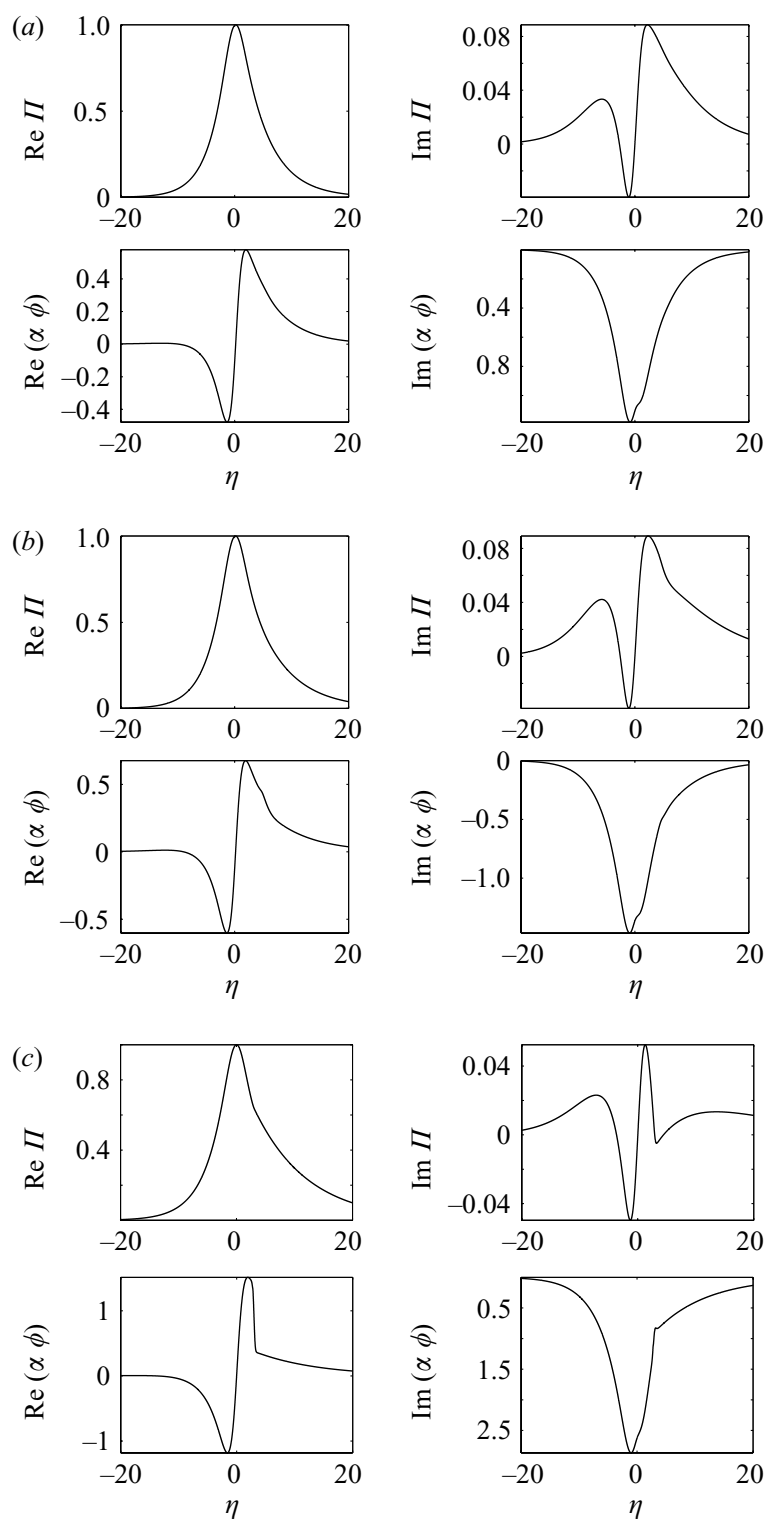

FIGURE 8 . Pressure and velocity eigenfunctions. The left-hand panels show the real part of the eigenfunction, while the right-hand panels show the complex part. (a) Hydrogen-oxygen-argon (b) hydrogen-oxygen-nitrogen and (c) propane systems.

the eigenfunction behaviour. The eigenfunctions for the low activation energy system differ only slightly from those for a frozen flow (not shown). For higher activation energies, the real part of the pressure perturbation becomes asymmetric with the cold side becoming the stronger side. In the same way, the magnitude of the normal velocity perturbation increases with an increase in activation energy. For the normal velocity, the low and medium activation energy cases are similar. The fresh side, $\eta>0$, eigenfunctions for the high activation energy case differ significantly from the two other cases. The region where anomalous behaviour is observed centres around $\eta=2.94$, which is the position of the transversal ignition point (evaluated as the 
location of the maximum temperature derivative). This location corresponds to a dimensional distance of $0.089 \mathrm{~mm}$ from the shear-layer interface, and is of the same order of magnitude as the value based on the one-dimensional analysis of $\$ 3.2$, that is, $0.064 \mathrm{~mm}$.

\subsection{Finite-rate chemistry effect}

The importance of finite-rate chemistry in the perturbation equation is determined by performing a new set of computations where the mean flow is defined by the reactive Navier-Stokes equations, (4.1), while the perturbation evolution, cf. (4.14), is governed by the chemically frozen counterpart. This analysis is focused on the propane system. For a frozen flow, the pressure perturbation equation coefficients analogous to those in (4.24) are (Criminale et al. 2003),

$$
\begin{aligned}
B & =-2 \frac{\bar{u}^{\prime}}{\bar{u}-c}, \\
C & =-\alpha^{2} \bar{T}^{2}\left[1-M^{2}(\bar{u}-c)^{2} / \bar{T}\right] .
\end{aligned}
$$

A comparison between the growth rate for non-reactive and reactive perturbation is shown in figure $9(a)$. This figure shows that chemical reaction in the perturbation evolution equation has a negligible effect on the growth rate. This observation points to the mean flow as the principal factor in determining the linear growth-rate attenuation linked to an increase in effective activation energy.

The eigenfunctions that correspond to the maximum growth rate are shown in figure $9(b)$, where frozen and reactive eigenfunctions are compared. The location of the ignition front is identified with that of the maximum mean-flow temperature derivative and is marked by the dash-dotted vertical lines in the figure $9(b)$. The frozen eigenfunctions differ significantly from the reactive ones, most markedly in the cold stream. Moreover, the frozen eigenfunctions are qualitatively similar to those for the hydrogen-oxygen-nitrogen system shown in figure $8(b)$.

\subsection{Energy balance in the shear layer}

More insight into the role of the transversal ignition front on the perturbation dynamics can be gained by analysing the energy transfer between the mean flow and perturbations. We shall compare the terms based on the inviscid perturbation analysis to terms based on viscous dissipation, in order to prove that they are small. For this comparison, the viscous dissipation terms are computed on the basis of the inviscid eigenfunctions. We start by defining the kinetic energy $K$ of the fluctuation as follows:

$$
K \equiv\left(\int_{-y}^{y} \int_{x}^{x+2 \pi /\left|\alpha_{r}\right|} \int_{t}^{t+2 \pi /|\omega|} \bar{\rho} \frac{\tilde{\hat{u}} \hat{\boldsymbol{u}}}{2} \mathrm{~d} t \mathrm{~d} x \mathrm{~d} y\right) / \frac{4 \pi^{2}}{\left|\alpha_{r} \omega\right|},
$$

where $\alpha_{r}$ is the real part of $\alpha$, the hat denotes perturbation quantities, i.e. the second term in the right-hand side of (4.13), and the tilde denotes complex conjugate. The material derivative based on the most unstable eigenvalue analysis is,

$$
\begin{aligned}
\frac{\mathrm{d} K}{\mathrm{~d} t}= & \operatorname{Re}\left\{\int_{-\eta}^{\eta}(\underbrace{-\tilde{f} \alpha \phi \bar{\rho}^{2} \bar{u}^{\prime}}_{T_{1}}+\underbrace{\frac{-\mathrm{i} \tilde{\alpha} \tilde{f} \Pi+\bar{\rho} \tilde{\alpha} \tilde{\phi}^{\prime} \Pi}{\gamma_{\infty} M^{2}}}_{T_{2}}+\underbrace{\frac{2 \alpha_{i} \tilde{f} \Pi-\bar{\rho}(\tilde{\alpha} \tilde{\phi} \Pi)^{\prime}}{\gamma_{\infty} M^{2}}}_{T_{3}} \frac{\mathrm{d} \eta}{\bar{\rho}}\}\right. \\
& \times \frac{1-\exp \left(-4 \pi\left(\alpha_{i} /\left|\alpha_{r}\right|\right)\right)}{4 \pi\left(\alpha_{i} /\left|\alpha_{r}\right|\right) \exp \left(2 \alpha_{i} x\right)} .
\end{aligned}
$$



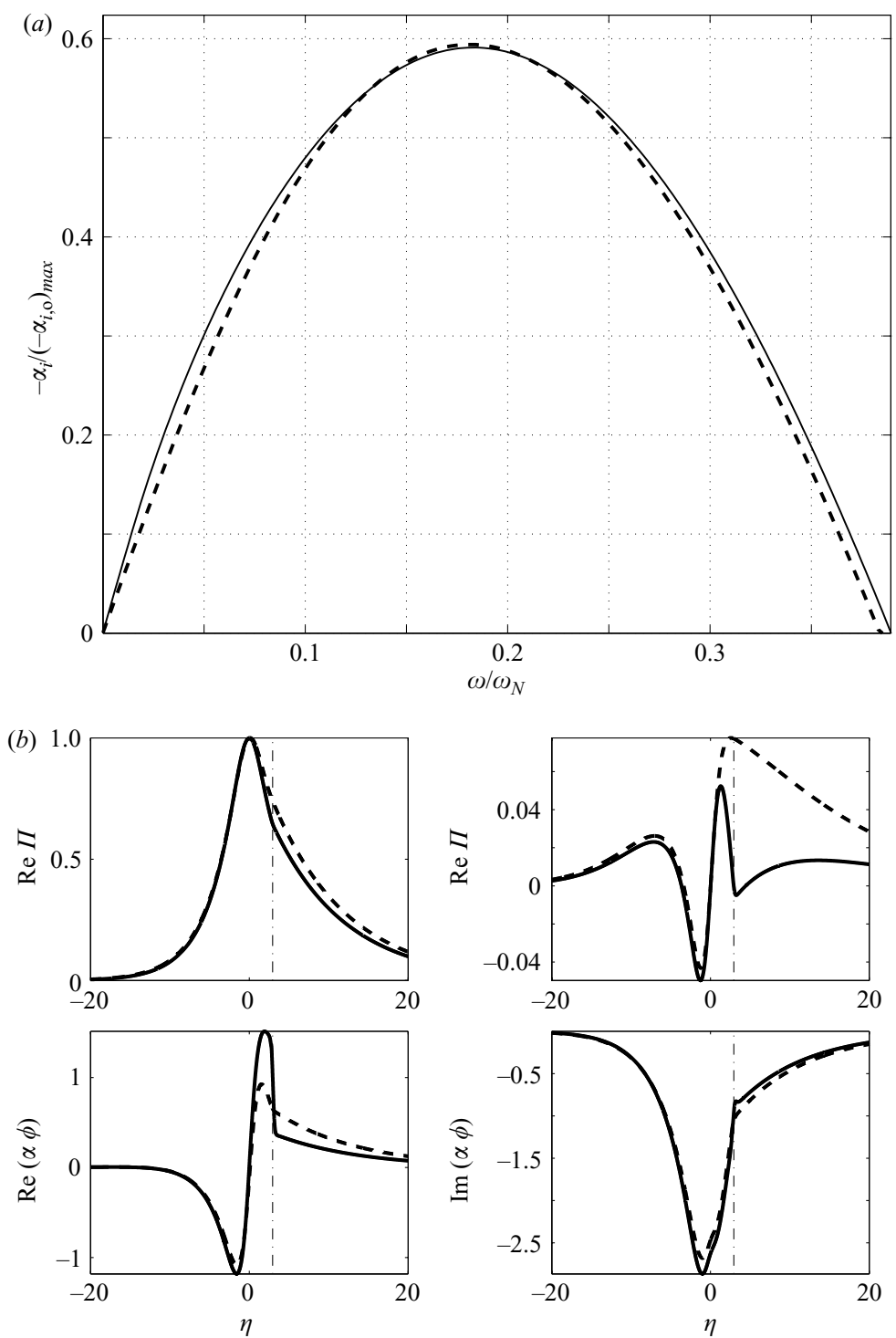

FIGURE 9. Solutions of chemically frozen and reactive perturbation evolution equations for a given mean flow of the propane system. The solid line is the reactive case while the dashed line is the frozen case. (a) Spatial growth rate. $(b)$ Eigenfunctions. The vertical dash-dotted line represents the location of the maximum mean-flow temperature derivative, located at $\eta=2.94$.

We note that each term, $T_{1-3}$, is a function of $\eta$ only, while the integral in (4.30) is operated over the physical non-dimensional coordinate $y$. Dissipative effects in the perturbation dynamics are related to an extra term that would be part of the integral operand in (4.31), namely,

$T_{4} \equiv \mathrm{i} \tilde{\alpha} \tilde{f} s_{x x}-\bar{\rho} \tilde{f}^{\prime} s_{x y}+\mathrm{i} \tilde{\alpha}^{2} \tilde{\phi} s_{x y}-\bar{\rho} \tilde{\alpha} \tilde{\phi}^{\prime} s_{y y}-2 \alpha_{i}\left(\tilde{f} s_{x x}+\tilde{\alpha} \tilde{\phi} s_{x y}\right)+\bar{\rho}\left(\tilde{f} s_{x y}+\tilde{\alpha} \tilde{\phi} s_{y y}\right)^{\prime}$,

where,

$$
\begin{gathered}
\Delta \equiv-2 / 3\left(\mathrm{i} \alpha f+\bar{\rho} \alpha \phi^{\prime}\right) \\
s_{x x} \equiv \frac{\mu}{\operatorname{Re}}(2 \mathrm{i} \alpha f+\Delta), \quad s_{y y} \equiv \frac{\mu}{\operatorname{Re}}\left(2 \bar{\rho} \alpha \phi^{\prime}+\Delta\right), \quad s_{x y} \equiv \frac{\mu}{\operatorname{Re}}\left(\bar{\rho} f^{\prime}+\mathrm{i} \alpha^{2} \phi\right)+\frac{m}{\operatorname{Re}} \bar{\rho} \bar{u}^{\prime},
\end{gathered}
$$



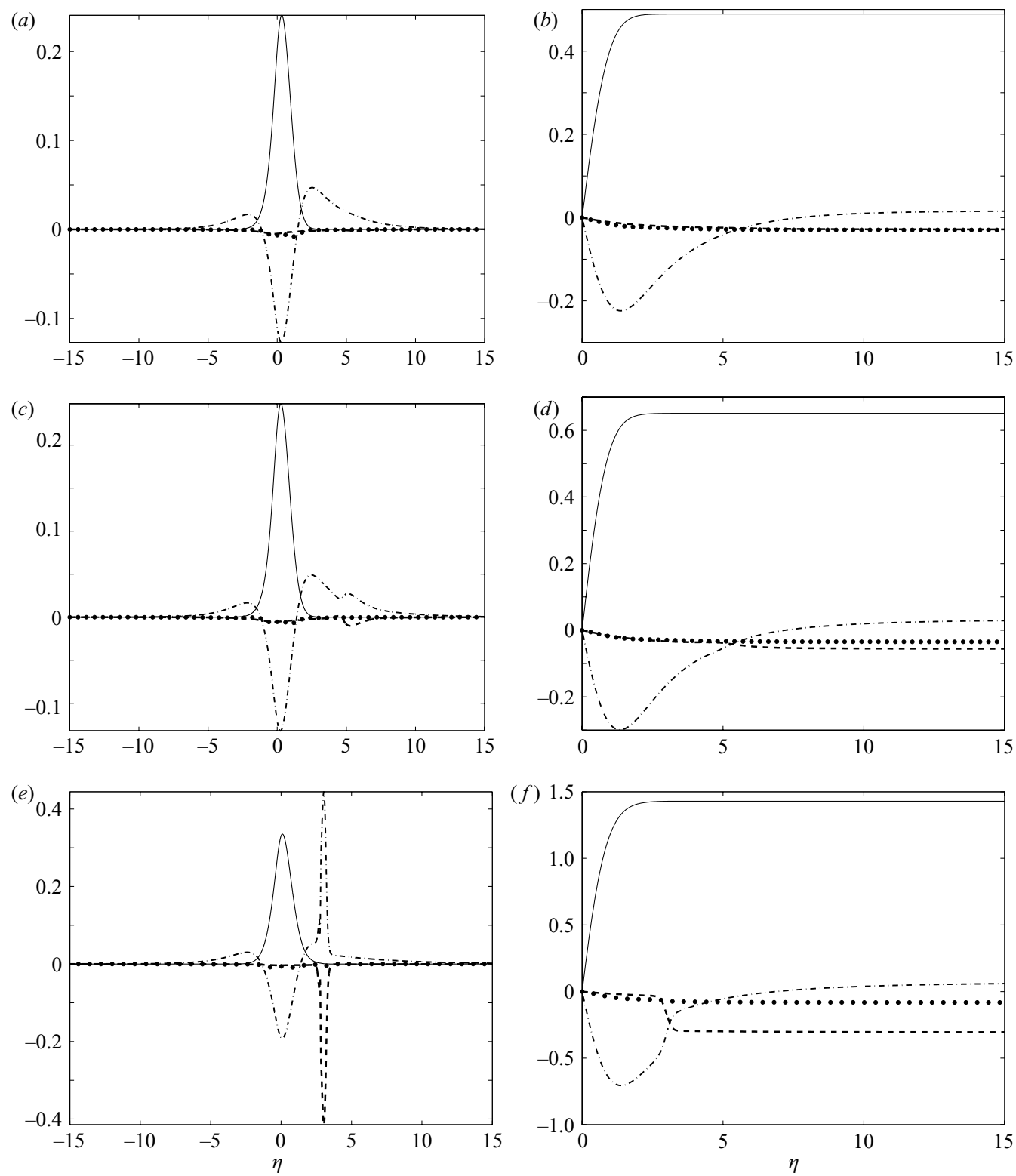

FIGURE 10. Energy balance in the shear layer. The solid, dashed and dash-dotted lines represent terms $T_{1-3}$ in (4.31), respectively, while the filled circles represent $T_{4}$, equation (4.32). $(a, b)$ Hydrogen-oxygen-argon, $(c, d)$ hydrogen-oxygen-nitrogen and $(e, f)$ propane systems. $(b, d, f)$ as $(a, c, e)$, integrated over $y$.

and $\mu$ and $m$ are the (non-dimensional) shear viscosity and its perturbation, respectively.

The aforementioned terms, $T_{1-4}$, are plotted in figure 10 versus $\eta$ with solid, dashed and dash-dotted lines for terms $T_{1-3}$, respectively, and with filled circles for term $T_{4} . T_{3}$ is essentially a transfer term, representing pressure velocity correlations at the boundary. Its integrated value is small because the normal contribution approaches zero as $\eta \rightarrow \infty$ where the perturbation vanishes, while streamwise contributions are generally small. $T_{1}$ is a Reynolds stress mean strain correlation and is the term 
responsible for the largest energy exchange between mean flow and perturbation. The term does not change significantly for the three mixtures. $T_{2}$ is a pressure-strain correlation term. Its magnitude is very different among the three mixtures: in the hydrogen-oxygen-nitrogen and hydrogen-oxygen-argon cases it is everywhere small, while in the propane case it is significantly larger. The term is maximum in absolute value close to the transversal ignition front (figure $10 c, e$ ), and its overall effect is to diminish the energy exchange between mean-flow and perturbation (figure $10 d$ ). The overall contribution of viscous dissipation is small everywhere, as shown by the filled circles in all parts of figure 10. The analysis therefore suggests that the decrease in growth rate associated with an increase in activation energy is linked to pressurestrain correlations at the ignition front. Stronger transversal fronts, i.e. thinner flames, support a stronger correlation.

The linear analysis leads us to the conclusion that the correlation between growthrate reduction and increasing effective activation energy centres around the decoupling between dynamic shear and thermal gradient in the mean profile. The dynamic shear region supports a large energy transfer between mean flow and perturbation owing to the Reynolds stress-mean profile correlation, while the ignition front supports a minimum in the pressure-strain correlation where energy is transferred from the perturbation to the mean flow. The decoupling is associated with the transversal ignition front propagation in the premixed mixture, a feature that is marked only for large activation energy mixtures, cf. §3.2.

Day, Reynolds \& Mansour (1998) related the attenuation of the disturbance growth in diffusion-controlled reactive mixing layers to the reduction of the density-weighted vorticity, $\bar{\rho} \mathrm{d} \bar{u} /\left.\mathrm{d} \eta\right|_{\eta=0}$. It should be noted that in Day et al. (1998), a flame sheet model was used so that the effect of the effective activation energy was not included. For the detonation triple-point shear layer, the value of the density-weighted vorticity is decreased by a decrease in the transversal flame thickness, cf. figure $4(d)$, and therefore, for the cases examined here, a correlation between $\bar{\rho} \mathrm{d} \bar{u} /\left.\mathrm{d} \eta\right|_{\eta=0}$ and mixture activation energy can be established. Thus, the mechanism of spatial growth attenuation in detonation triple-point shear layers is similar to those described in earlier work on diffusion-limited combustion systems, but here the mixture activation energy is a controlling factor by means of its effect on the flame thickness. The medium and low counterparts have similar shear-layer characteristics, even though their mixture properties are different. These concepts will be elaborated on by performing twodimensional Navier-Stokes simulations on the systems. The relation between shearlayer instability and thermal runaway in the premixed streams will be the focal point of the nonlinear analysis, with the goal of elucidating the cause of the localized explosions in the experiments.

\section{Nonlinear numerical analysis}

Three principal phenomena occur in the evolution of the triple-point shear layer: the Kelvin-Helmholtz instability, the auto-ignition of the shocked unreacted stream, and the transversal front propagation. The relation between the time scales of the phenomena probably controls their interaction. In the linear analysis, $\S 4$, the length scale of the instability is identified with the inverse of the spatial eigenvalue, $\alpha$ : the high activation energy mixture has a smaller eigenvalue and therefore the instability develops at larger distances from the triple point. The relation between instability length scale and induction distance is important because for the convective transport associated with the instability to produce localized ignition, structures have to form 
upstream of the adiabatic induction distance. As a result, linear analysis is concerned with events preceding auto-ignition and it is limited to the region close to the triple point. Nonetheless, a nonlinear fluid dynamic analysis focused on the interaction among induction, instability and transversal ignition is important for investigating the formation of 'hotspot' and localized explosion in the unreacted mixture. The nonlinear analysis is carried out through numerical solutions of the two-dimensional Navier-Stokes equations.

The set of governing equations is

$$
\frac{\partial \boldsymbol{U}}{\partial t}+\frac{\partial \boldsymbol{F}}{\partial x}+\frac{\partial \boldsymbol{G}}{\partial y}=\dot{\omega},
$$

where:

$$
\begin{aligned}
\boldsymbol{U}=\left[\begin{array}{l}
\rho \\
\rho u \\
\rho v \\
\rho e_{0} \\
\rho Y_{i}
\end{array}\right], \quad \boldsymbol{F}=\left[\begin{array}{l}
\rho u \\
\rho u^{2}-\sigma_{x x} \\
\rho u v-\sigma_{y x} \\
\left(\rho e_{0}-\sigma_{x x}\right) u-\sigma_{x y} v-\lambda \frac{\partial T}{\partial x}-\sum_{k}\left(\sum_{i} v_{i, k} h_{i}\right) \frac{\partial Y_{k}}{\partial x} \\
\rho u Y_{i}+\sum_{k} v_{i, k} \frac{\partial Y_{k}}{\partial x}
\end{array}\right], \\
\boldsymbol{G}=\left[\begin{array}{l}
\rho v \\
\rho u v-\sigma_{x y} \\
\rho v^{2}-\sigma_{y y} \\
\left(\rho e_{0}-\sigma_{y y}\right) v-\sigma_{y x} u-\lambda \frac{\partial T}{\partial y}-\sum_{k}\left(\sum_{i} v_{i, k} h_{i}\right) \frac{\partial Y_{k}}{\partial y} \\
\rho v Y_{i}-\sum_{k} v_{i, k} \frac{\partial Y_{k}}{\partial y} .
\end{array}\right] .
\end{aligned}
$$

Here, $\sigma$ is the stress tensor and all the summations extend to all species. Unlike in the linear analysis where Lock's approximation was used, (4.6), the mixture average shear viscosity is found by using a kinetic theory approximate solution (see Dixon-Lewis 1968), as for the other transport coefficients.

The Navier-Stokes equations are discretized using a third-order in time, fourthorder in space Rusanov, Kutler, Lomax and Warming (RKLW) scheme described in Kennedy \& Carpenter $(1997,1994)$. For a linear constant coefficient scalar equation in one dimension, the fourth-order scheme is stable with a maximum CFL condition of 1.34. Even so, the RKLW scheme is not monotone or total variation diminishing (TVD), thus, in order to preserve stability for nonlinear or variable coefficient equations, linear filtering is used to damp out spurious oscillations. A fourth-order explicit filter, also discussed in Kennedy \& Carpenter (1997), is used.

Stiff chemistry is handled using a fractional step-splitting technique. The splitting considers the time integration of the transport and kinetic parts of the NS equations separately. The splitting scheme preserves the spatial order of the discretization, but lessens the temporal order of the RKLW, producing a second-order time discretization. The system of governing equations is written in the operator form,

$$
\frac{\partial \boldsymbol{Q}}{\partial t}=\mathscr{T} \boldsymbol{Q}+\mathscr{K} \boldsymbol{Q},
$$




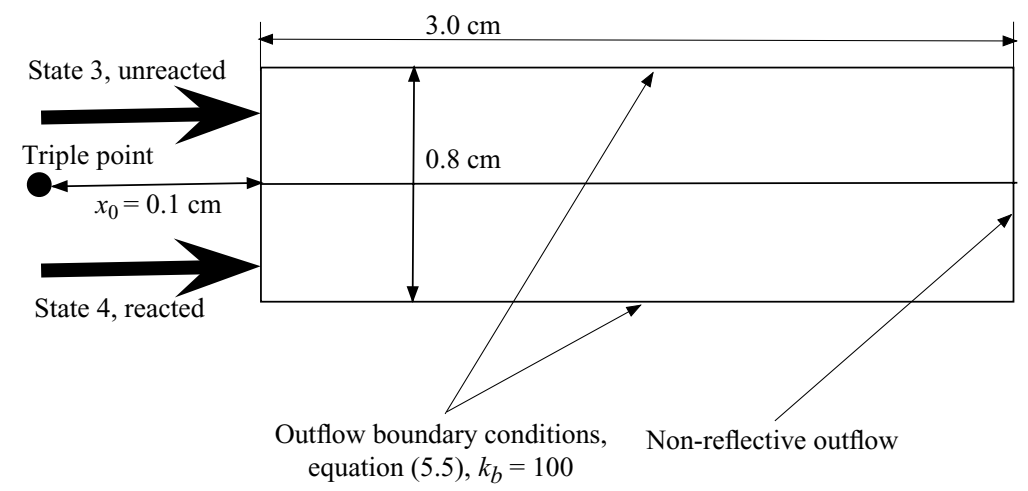

FIGURE 11. Sketch of the computational domain and boundary conditions, not to scale.

where the field variable is $\boldsymbol{Q}$, and $\mathscr{T}$ and $\mathscr{K}$ are the transport and kinetic operators, respectively. Consider the two discrete solution operators, $\mathscr{T}^{n}(\Delta t)$ and $\mathscr{K}^{n}(\Delta t)$ which approximate the solutions at time $t_{0}+\Delta t$ to the problems in which only one operator at a time is considered. The overall update is

$$
\boldsymbol{Q}^{n+1}=\mathscr{T}^{n+1}\left(\frac{\Delta t}{2}\right) \mathscr{K}^{n+1 / 2}(\Delta t) \mathscr{T}^{n}\left(\frac{\Delta t}{2}\right) \boldsymbol{Q}^{n},
$$

where the superscript $n$ denotes the level at which the independent variable, time, is considered. Assuming that both $\mathscr{T}^{n}(\Delta t)$ and $\mathscr{K}^{n}(\Delta t)$ yield an approximation of order $(2,4)$ to the partial problems, the numerical solution to the Navier-Stokes equations is accurate to order $(2,4)$. The kinetic update is a point-implicit operation and is discretized using the Crank-Nicolson formula. The kinetic update involves progress along the reaction paths as well as the imposition of elemental mass conservation. Given that the latter constitutes a linear time-independent coefficient system, the associated matrix is inverted a priori, i.e. within the initialization procedure. Chemistry update via the Crank-Nicolson method involves, therefore, the solution of a $N_{s p}-N_{e l}$ system of equations, where $N_{e l}$ is the number of atomic elements.

\subsection{Boundary conditions}

The boundary conditions and the dimensions of the computational domain are sketched in figure 11. Inflow boundary conditions for the non-linear analysis are found from the exact solution to the non-reactive boundary-layer equations in selfsimilar form, equation (4.12). The separation distance from the point of first mixing is assumed to be $x_{0}=0.1 \mathrm{~cm}$. Issues concerning the size of the domain, the boundary conditions and the effect of the variation of $x_{0}$ on the solution are discussed in more detail in $\S 5.1 .2$.

Neglecting diffusive terms, boundary conditions are obtained from a characteristic variable decomposition of the reactive Euler equations and a local one-dimensional inviscid analysis (LODI) in the direction normal to the boundary (Poinsot \& Lele 1992). The incoming characteristics are determined from

$$
\frac{\partial \boldsymbol{P}}{\partial t}=\mathscr{P} \frac{\partial \boldsymbol{Q}}{\partial t}=k_{b}\left(\boldsymbol{P}_{b}(t)-\boldsymbol{P}\right)
$$

where $k_{b}$ is a parameter, and the matrix $\mathscr{P}$ is a rectangular matrix of size $\left[m \times N_{s p}+4\right]$ having as rows those of the Jacobian $\partial \boldsymbol{P} / \partial \boldsymbol{Q}$ corresponding to the $m$ imposed primitive variables $\boldsymbol{P}_{b}$. Considering a boundary with constant streamwise coordinate, 
$x$, multiplying the Navier-Stokes equations by $\mathscr{P}$, we obtain

$$
k_{b}\left(\boldsymbol{P}_{b}-\boldsymbol{P}\right)=-\mathscr{P}\left(\frac{\partial \boldsymbol{F}}{\partial x}+\frac{\partial \boldsymbol{G}}{\partial y}-\dot{\omega}\right),
$$

and neglecting the viscous fluxes in the direction normal to the boundary, a spectral decomposition of the flux Jacobian yields

$$
\frac{\partial \boldsymbol{F}}{\partial x}=R^{-1} \Lambda R \frac{\partial \boldsymbol{Q}}{\partial x}=R^{-1} \mathscr{L} .
$$

Substituting (5.7) into (5.6), we obtain a linear system with associated coefficient matrix of size $m \times N_{s p}+4$ in the unknown vector $\mathscr{L}$. The coefficient matrix is $\mathscr{M}=\mathscr{P} R^{-1}$. This system is augmented with the evaluation of the wave amplitudes, elements of $\mathscr{L}$, associated with outgoing waves using one-sided third-order finitedifference approximation about the boundary point. Once the wave amplitudes are solved for, (5.7) is used to evaluate the fluxes normal to the boundary. For nonreflecting boundary conditions, the columns of $\mathscr{M}$ corresponding to the outgoing waves are zeroed.

For the simulations presented below, reflective conditions are applied to the inflow, where, on the subsonic side, all the primitive variables except pressure are imposed, and non-reflecting boundary conditions with imposed pressure are applied to the outflow boundaries and the side boundaries. The injection conditions are perturbed by adding a white noise (uniformly random and uncorrelated) disturbance on the imposed streamwise velocity. The magnitude of the disturbance has been selected at $4 \%$ of the nominal injection value. A set of runs with different white noise magnitudes have revealed insensitivity of the ignition-instability interaction to this parameter.

For a non-reflective boundary condition, the constant $k_{b}$ in (5.6), represents an elastic response of the outside system to perturbation of the boundary values. The term non-reflective indicates that the response of the system to outgoing waves is neglected. When the constant $k_{b}$ is set equal to zero, a perfectly non-reflective boundary condition, in the sense of Poinsot \& Lele (1992), is recovered, while for $k_{b} \rightarrow \infty$ a constant-pressure boundary is obtained. The role of $k_{b}$ in our shear-layer simulations is discussed in more detail in $\S 5.1 .2$.

\subsubsection{One-dimensional analysis of the cold/supersonic stream}

An Euler one-dimensional reactive analysis for the cold stream is of interest because the associated injection conditions are supersonic and therefore a classical ZND detonation structure is not supported. The flow becomes sonic before chemical equilibrium is reached, resulting in a singularity. This outcome is a necessary consequence of the non-existence of equilibrium solutions for the given (injection) values of mass flow, momentum flux, total enthalpy and atomic fluxes, $(\rho u)_{0},\left(p+\rho u^{2}\right)_{0}, h_{0}^{o}$ and $\dot{N}_{O, H, C, N} \equiv \rho u N_{O, H, C, N}$; here $N$ is the mole number. Equilibrium one-dimensional non-diffusive solutions are determined from the relations,

$$
\begin{aligned}
h^{o} & =\left(h^{o}\right)_{0}, \\
\rho u & =(\rho u)_{0}, \\
P+\rho u^{2} & =\left(P+\rho u^{2}\right)_{0}, \\
\dot{N}_{O, H, C, N} & =\left(\dot{N}_{O, H, C, N}\right)_{0}, \\
\dot{w}_{i=1: N_{s}}-N_{e l} & =0 .
\end{aligned}
$$



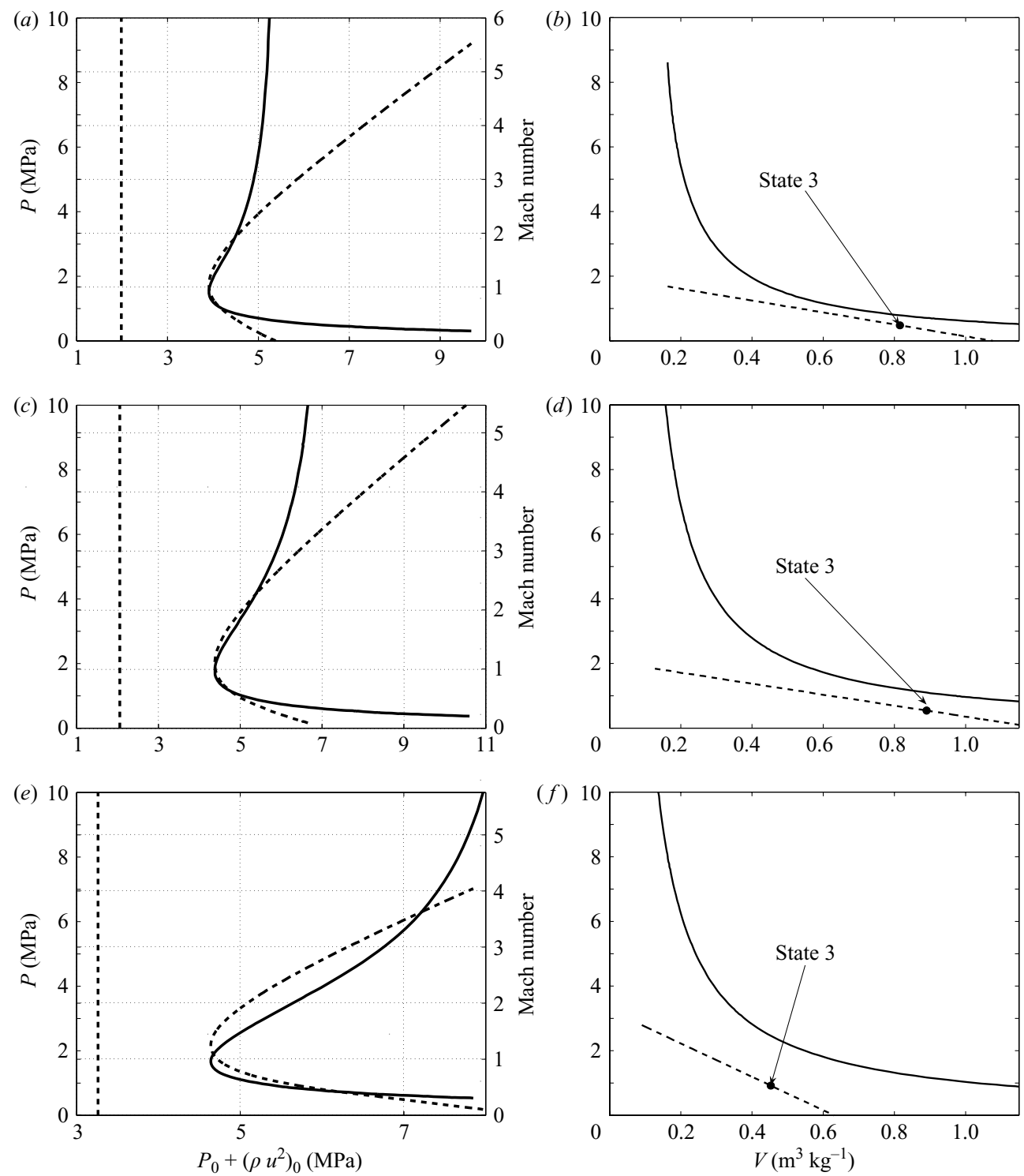

FIGURE 12. Equilibrium solutions for $(a, b)$ hydrogen-oxygen-argon, $(c, d)$ hydrogen-oxygen-nitrogen and $(e, f)$ propane systems. $(a, c, e)$ The dashed line shows pressure and the solid line Mach number. The vertical dashed lines are the shock-polar momentum fluxes. $(b, d, f)$ The solid line is the product Hugoniot curve and the dashed line is the Rayleigh line.

In figure 12(a,c,e) the equilibrium values for pressure and Mach number are plotted against the momentum flux for the three mixtures. The lines correspond to the case for which the total enthalpy, mass flux and the molar atomic fluxes are equal to the 'nominal' values referred to as state 3 in the triple-point shock-polar (figure $2 c$ ) and in table 1 . The vertical dashed lines indicate the nominal momentum flux. The lack of an equilibrium solution is associated with the presence of a turning point in correspondence of the sonic condition, so that a limiting value of the momentum flux is defined for each system and no intersection occurs with the state 3 values. 
(The sonic conditions are based on the frozen speed of sound. The turning point is therefore not located exactly at $M=1$, which would be true for a constant properties mixture.) The same argument can be made from the pressure-specific volume, $P-V$, diagrams (figure $12 b, d, f$ ). In these diagrams the Rayleigh lines emanating from state $3, P-P_{3}=(\rho u)_{0}^{2}\left(V_{3}-V\right)$, and the Hugoniot curve for the equilibrium products, $h-h_{3}=1 / 2\left(P-P_{3}\right)\left(V+V_{3}\right)$, are shown. The lack of an equilibrium point is demonstrated by the two curves not having an intersection point.

We remark that this conclusion is valid only for a one-dimensional problem assuming adiabatic conditions, which is relevant to the shear-layer system if the curvature of the leading shock can be neglected and the distance from the hot/reacted stream is large enough. Experimental schlieren images show that the curvature of the leading shocks in the vicinity of the triple point is modest near the end of the cell cycle; a discussion on the dimensions of the shear-layer domain is to follow. Another phenomenon that we shall consider is the normal expansion of the stream, here identified with the stream tube divergence. Normal expansion enables a stationary solution when a one-dimensional wave solution is impossible. Assuming a quasi-onedimensional flow, the pressure differential is given by

$$
\mathrm{d} p=\frac{\rho u^{2}}{1-M^{2}}\left[\mathrm{~d}(\log A)+\sum_{k=1}^{N_{s}}\left(\frac{h_{k}}{c_{p} T}-\frac{R_{k}}{R}\right) \mathrm{d} Y_{k}\right],
$$

from which it is seen that stream tube divergence represented by the term $\mathrm{d}(\log A)$ ( $A$ is the cross-sectional area) can make the pressure derivative finite even when the thermicity, the second term in the square brackets, does not vanish at the sonic condition. The normal expansion can be quantified by the rate of change of the cross-sectional area delimited by pathlines,

$$
\delta_{a}=\frac{1}{A} \frac{\mathrm{D} A}{\mathrm{D} t}
$$

which in two dimensions is evaluated as

$$
\delta_{a}=\frac{\partial v_{n}}{\partial n}=(\nabla \boldsymbol{v} \cdot \boldsymbol{n}) \cdot \boldsymbol{n},
$$

where $\boldsymbol{n}=(-v, u) /\|\boldsymbol{v}\|$ is the normal to the streamline.

For a free shear layer the relation between $\delta_{a}$ and the (volume) dilatation

$$
\delta_{v}=\frac{1}{V} \frac{\mathrm{D} V}{\mathrm{D} t}
$$

is of particular importance in the region of high chemical activity. When thermal runaway occurs, the dilation increases sharply owing to the contribution of heat release,

$$
\Theta=-\sum_{k=1}^{N_{s}}\left(\frac{h_{k}}{c_{p} T}-\frac{R_{k}}{R}\right) \frac{\omega_{k}}{\rho},
$$

the streams expand, fluid leaves the domain, and outflow occurs at the side boundaries. Figure 12 shows that a one-dimensional steady inviscid solution is impossible for zero cross-sections area expansion, $(1 / A)(\mathrm{d} A / \mathrm{d} t)=0$, yet a constant-pressure inviscid steady solution is always possible; in such cases, the streamwise acceleration is zero because the area expansion contribution balances out the thermicity contribution.

In order to account for the unconstrained nature of the side boundaries and allow stream-tube expansion, the parameter $k_{b}$ in (5.5) for the side boundary conditions 

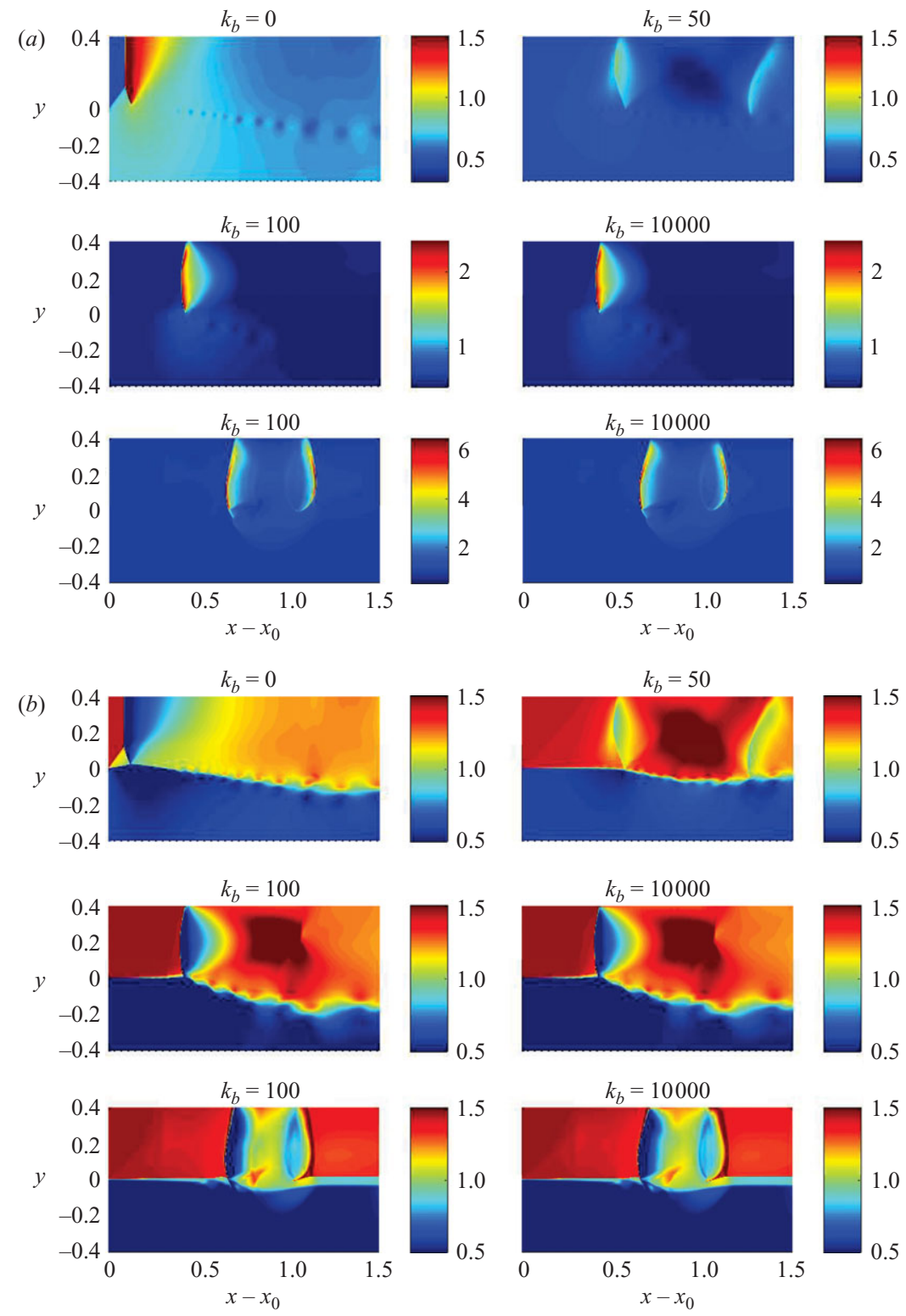

Figure $13(a, b)$. For caption see facing page.

has been increased from zero in order to simulate the response of the outer system to explosion in the streams. Obviously, the dimension of the solution domain in a direction perpendicular to the flow has an effect on these conclusions, as discussed in more detail in the following section.

\subsubsection{Analysis of side boundary conditions}

In this section, we discuss a series of issues related to the boundary conditions and the dimension of the computational domain. The main focus is on the physical extent of the shear layer in the direction perpendicular to the streamwise direction and, in particular, the dimension of the unreacted stream, labelled as state 3 in figure $2(c), h_{3}$. 

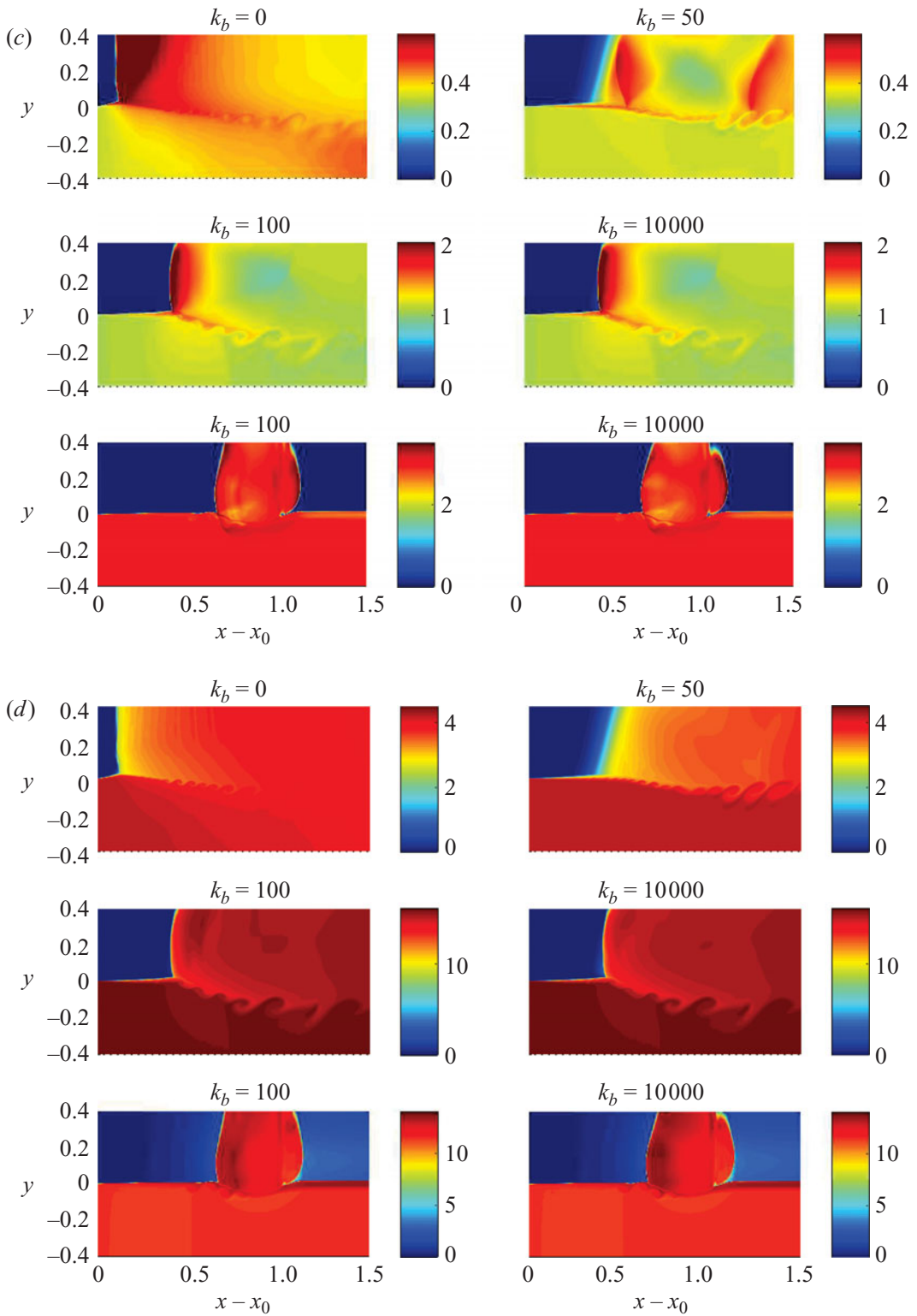

FiguRE 13. Numerical solution for different values of $k_{b}$ in (5.5). The first row of plots in each part refers to the hydrogen-oxygen-argon system at $45 \mu \mathrm{s}$, the second row to the hydrogenoxygen-nitrogen system at $15 \mu \mathrm{s}$, the third to the propane system at $9.5 \mu \mathrm{s}$. (a) Pressure, MPa. (b) Mach number. (c) $\mathrm{OH}$ mass fraction, $\times 100$. (d) $\mathrm{H}_{2} \mathrm{O}$ mass fraction, $\times 100$.

Examining the reaction zone keystone structure (Pintgen et al. 2003b), it is evident that the maximum value of $h_{3}$ occurs at the inflow boundary of the shear layer and is equal to the induction distance behind the incident wave $\Delta_{t s} . h_{3}$ decreases monotonically to zero at the location of the auto-ignition point in the cold stream. Values of maximum $h_{3}$ are calculated as $h_{3}=w_{2} \times \tau_{2}$ where $w_{2}$ is the post-shock velocity and $\tau_{2}$ is the induction time, assuming a constant volume adiabatic explosion 


$\begin{array}{cccc}\text { Mixture } & w_{2}\left(\mathrm{~m} \mathrm{~s}^{-1}\right) & \tau_{2}(\mu \mathrm{s}) & \max . h_{3}(\mathrm{~cm}) \\ 2 \mathrm{H}_{2}-\mathrm{O}_{2}-17 \mathrm{Ar} & 253 & 8.9 & 0.2 \\ 2 \mathrm{H}_{2}-\mathrm{O}_{2}-5.6 \mathrm{~N}_{2} & 329 & 16 & 0.5 \\ \mathrm{C}_{3} \mathrm{H}_{8}-5 \mathrm{O}_{2}-9 \mathrm{~N}_{2} & 360 & 29 & 1.0\end{array}$

TABLE 2. Calculated maximum vertical dimension of the cold stream $h_{3}$ of the triple-point shear layers for the three mixtures analysed.
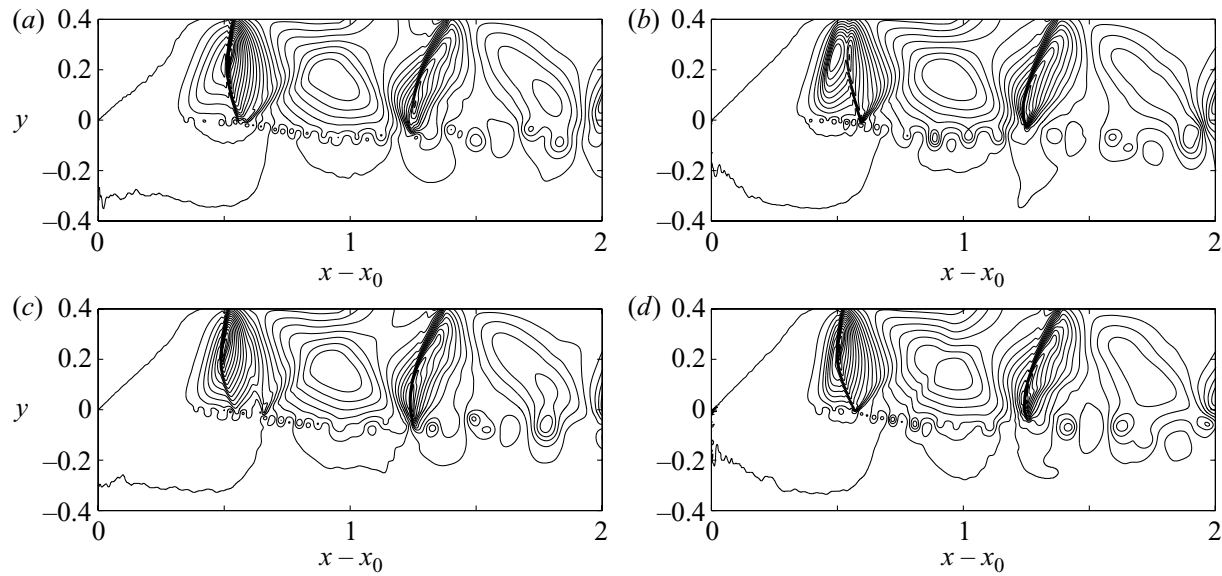

FIGURE 14. Pressure contours for the hydrogen-oxygen-argon system for $k_{b}=100$ at times (a) $85 \mu \mathrm{s},(b) 90 \mu \mathrm{s},(c) 95 \mu \mathrm{s},(d) 100 \mu \mathrm{s}$.

behind the incident wave propagating at $0.9 U_{C J}$. Values of maximum $h_{3}$ for the three mixtures are given in table 2.

In the nonlinear analysis, the height of the computational domain is fixed at $0.8 \mathrm{~cm}$ for the three mixtures, thus the simulated unreacted stream extends for $0.4 \mathrm{~cm}$ in the normal direction. Such a value is comparable to maximum $h_{3}$ for the hydrogenoxygen-argon and hydrogen-oxygen-nitrogen mixtures, but is lower than the corresponding value for the propane system; this assumption will be discussed at the end of the present section.

The influence of the side boundary conditions on the solution is analysed by varying $k_{b}$ in (5.5). The results of the study are summarized in figures 13 and 14; details on the computational meshes are provided in $\S 5.2 .1$. The analysis leads to the following observations.

(i) For the low activation energy system there exists a value $0<k_{b}<50$ for which the system transitions from one that supports a shock wave in the fresh mixture to one that supports a regular ignition structure. In particular, when $k_{b}=0$, a shock forms in the fresh mixture and propagates towards the inflow boundary, figure 13(a).

(ii) The low activation system reaches a quasi-stationary state away from the mixing region when $k_{b}>50$ (figure 14). Different values of $k_{b}$ above 50 do not significantly change the solution close to the mixing layer.

(iii) For the hydrogen-oxygen-nitrogen and propane mixtures, all values of $k_{b}$ lead to the formation of shock waves and consequent advection of the ignition to the whole unreacted stream. Changing the value of $k_{b}$ does not significantly change the solution close to the mixing layer. 

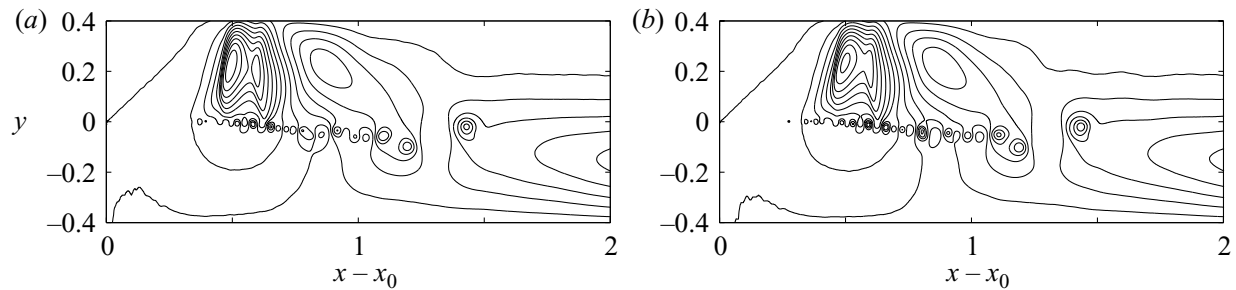

FIGURE 15. Pressure contours for the hydrogen-oxygen-argon system $150 \mu$ s for (a) $x_{0}=0.1 \mathrm{~cm},(b) 0.05 \mathrm{~cm}$.

(iv) In the case of the large activation energy system (propane system), two shock waves move upstream and downstream from the ignition point, while, in the two other mixtures, a single shock wave moves upstream (figure 13a). The different behaviour is because in the first cases ignition occurs close to the hot interface much faster than in the rest of the unreacted stream, while in the other cases (medium and low effective activation energy) auto-ignition occurs simultaneously over the top half of the shear layer (figure $13 c, d$ ).

A second issue regards the effect of the distance between the inflow boundary and the triple point, $x_{0}$, on the solution. Such an effect is found to be negligible, as demonstrated in figure 15. The figure shows a comparison between results obtained for the hydrogen-oxygen-argon system at $150 \mu$ s with two different values of $x_{0}, k_{b}$ was fixed at 100 .

Referring back to the discussion about the domain height at the beginning of this section, the results presented in figure 13 allow us to justify the choice of the computational domain for the propane system for two reasons. First, shocks form for any value of $k_{b}$, and enlarging the computational domain increases the likelihood of such an event. Secondly, the solution in the mixing region is not affected by the choice of $k_{b}$, and therefore boundary conditions do not affect the evolution at the shear-layer interface.

Although the interaction between the shear layer and the outer system is likely to be more complex when the entire detonation structure is considered, the simple boundary condition model provides a way to quantify the level of interaction between the free stream and the core flow. The fact that only for the hydrogen-oxygen-argon system can the flow be stabilized to form a regular explosion profile, provides an explanation for the formation of localized, intensely luminescent structures observed in high $\theta_{\text {eff }}$ mixtures. The system is unable to overcome the large volume increase due to reaction by means of stream-tube cross-sectional area dilatation.

The explanation for the formation of localized luminescent regions given in this section is obviously independent of the presence of vortical structures in the reactive stream before ignition. The interaction between the chemical processes and the fluid dynamic instability through convective thermal transport by shear-layer structures is the focus of the two-dimensional simulations.

\subsection{Two-dimensional results}

\subsubsection{Computational mesh}

The two-dimensional computational meshes are uniformly spaced in the streamwise direction with spacing $\mathrm{d} x=12.5 \mu \mathrm{m}$ and exponentially spaced in the direction perpendicular to the flow with minimum spacing $\mathrm{d} y_{\min }=1.7 \mu \mathrm{m}$ and average spacing $\mathrm{d} y_{\text {avg }}=20 \mu \mathrm{m}$. Using the length scale defined in $\S 4$ with $x=0.5 \mathrm{~cm}$, the minimum 

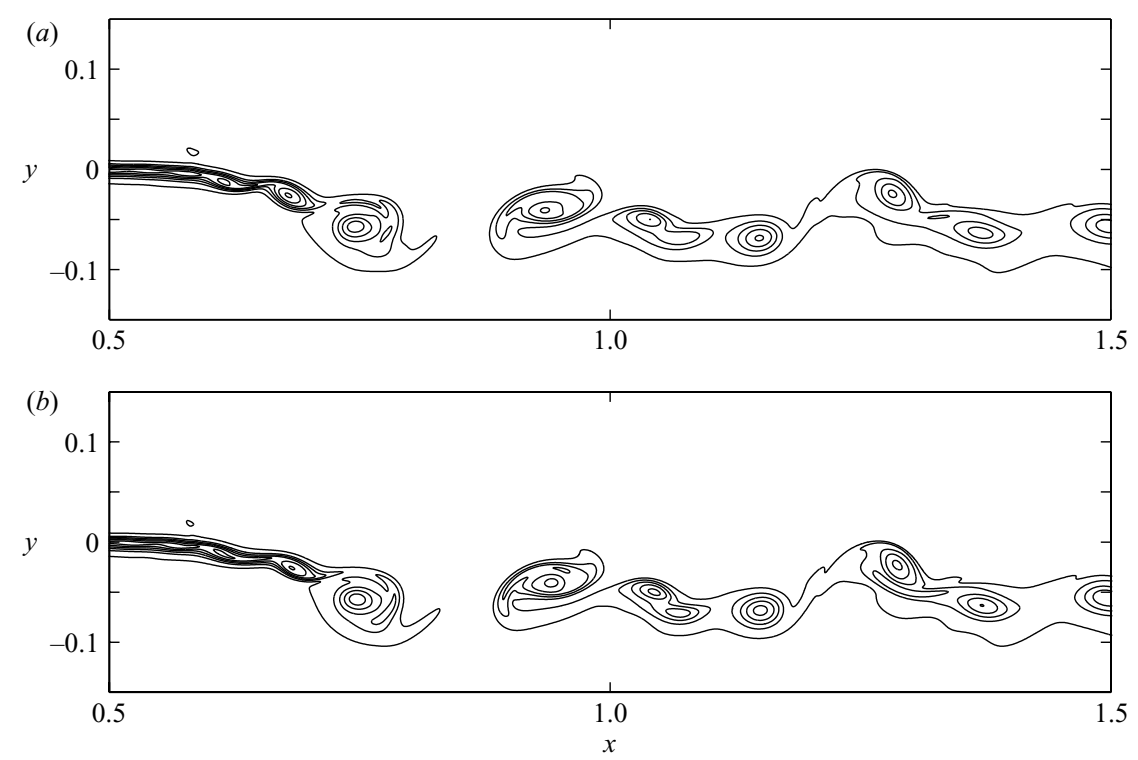

FIGURE 16. Resolution study. Vorticity, 30 contours in $[-3,0.01] \mu \mathrm{s}^{-1}$. The grids have a total number of mesh points of $(a) 2201 \times 375$ (coarse) and $(b) 3301 \times 565$ (fine).

spacing for the hydrogen-oxygen-argon system is, in non-dimensional units, $\mathrm{d} \bar{y}_{\text {min }}=7 \times 10^{-2}$.

A grid convergence study was performed by decreasing the spacings in both directions by a factor 1.5. The results of the grid refinement study are shown in figure 16. The figure shows vorticity contours for the hydrogen-oxygen-argon system and the comparison was performed by starting from the same interpolated condition at $t=150 \mu \mathrm{s}$, and integrating in time to $t=250 \mu \mathrm{s}$. The vortical structures shown in figures $16(a)$ and $16(b)$ have the same shape and location, with the highest vorticity contours slightly under-resolved by the coarse-grid computations. A possible source of disagreement between coarse and fine predictions can also be the randomness in the injection boundary velocity. All results presented in the next section are for the coarse mesh.

\subsubsection{Numerical experiments}

Numerical results are presented for the three mixtures. In all of the two-dimensional plots, the spatial dimensions are expressed in $\mathrm{cm}$. A snapshot of the solution field for the hydrogen-oxygen-argon system at time $30 \mu \mathrm{s}$ is presented in figure 17. This time scale corresponds approximately to the detonation cell cycle period for this mixture. The spatial development of the instability matches the experimental trends observed for the $2 \mathrm{H}_{2}-\mathrm{O}_{2}-7.7 \mathrm{~N}_{2}$ mixture in figure $2(b)$. The comparison between the aforementioned mixture and the hydrogen-oxygen-argon system is justified by the weak correlation between such parameter and the stability eigenvalue for low activation energy mixtures, cf. $\S 4$. A vortical structure of size $\approx 1.5 \mathrm{~mm}$ develops at a distance $\approx 5 \mathrm{~mm}$ from the point of first chemiluminescence. The unreactive triple point cannot be seen in the PLIF images, so a calculated induction distance behind the Mach stem (figure 2c) must be added to such distance. For state $4^{\prime}$ of $2 \mathrm{H}_{2}-\mathrm{O}_{2}-7.7 \mathrm{~N}_{2}$, the induction distance behind the Mach stem at the end of the cell cycle is approximately $1.8 \mathrm{~mm}$. The distance from the triple point at which 

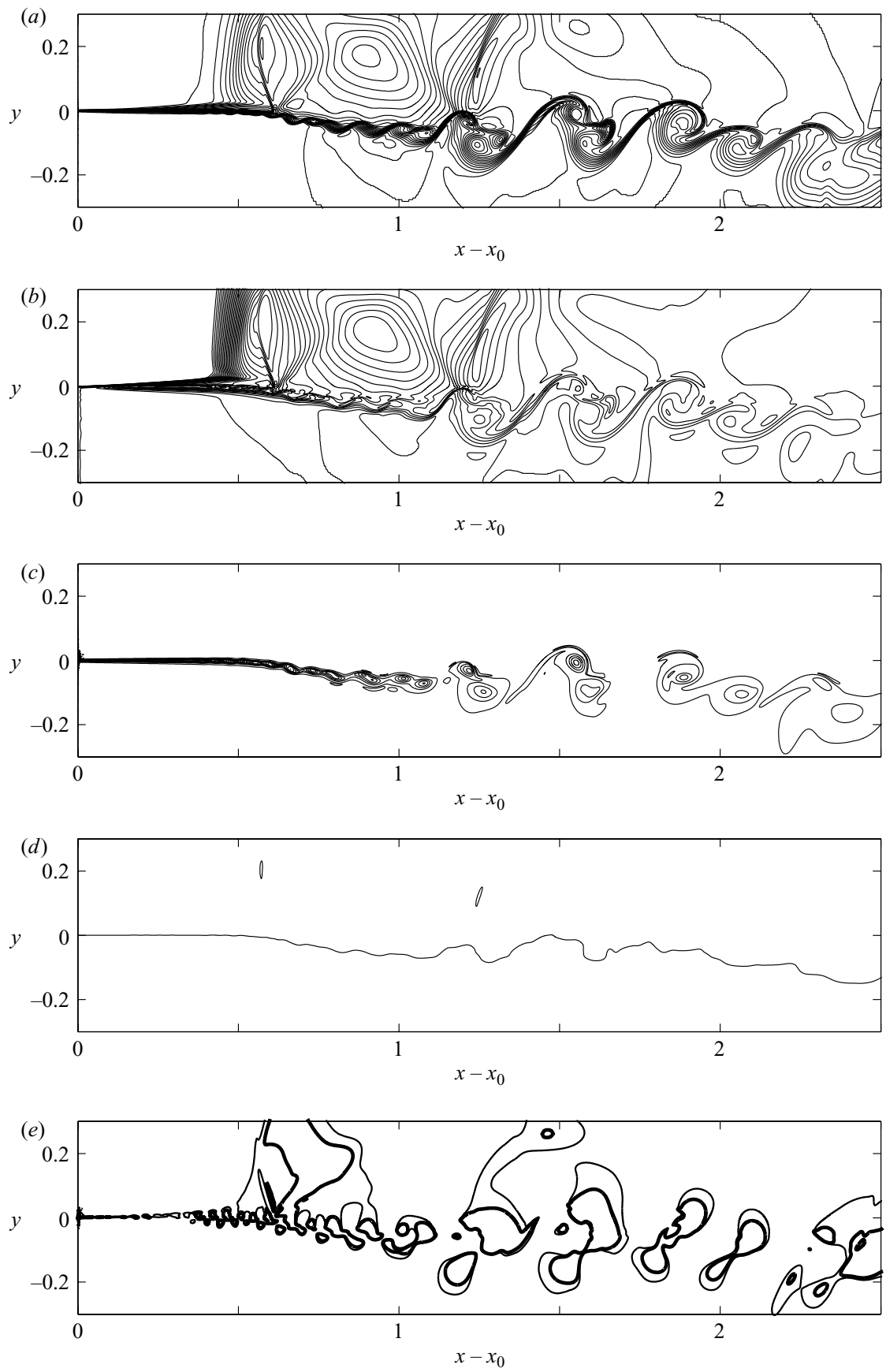

FIGURE 17. Numerical simulation of the hydrogen-oxygen-argon system shear layer. Distances are in $\mathrm{cm}$. (a) Temperature, 30 contours in $[1700,2800] \mathrm{K}$. (b) $\mathrm{OH}$ mass fraction, 30 contours in $[0,0.05]$. (c) Vorticity, 30 contours in $[-2.5,0.2] \mu \mathrm{s}^{-1}$. $(d)$ Mach number, one contour drawn for $\mathrm{Mach}=1.03$. (e) $\partial v_{n} / \partial n$, two contours: $0.05 \mu \mathrm{s}^{-1}$ thin line, and $0.1 \mu \mathrm{s}^{-1}$ thick line.

millimetre size structures are observed in the experiments is thus of the order of $7 \mathrm{~mm}$. Structures of the same magnitude are observed in the computation of figure 17 at a distance of $\approx 8 \mathrm{~mm}$ from the injection boundary. Figure $17(b)$ shows that ignition 
occurs at a distance of approximately $5 \mathrm{~mm}$ from the injection, a value close to the constant-volume induction distance $\left(u_{3} \tau_{3}=5.9 \mathrm{~mm}\right)$ based upon values given in table 1. Large-scale vortical structures develop downstream of the ignition point, so that the instability does not have a noticeable effect on thermal runaway. On the other hand, the ignition of the cold stream appears to slow down the formation and growth of vorticity structures. A similar phenomenon was noted by Hermanson \& Dimotakis (1989).

Figure $17(d)$ shows the Mach number, $M$. The Mach number in the cold/unreacted stream is everywhere greater than one. The single contour $M=1.03$ shows the lowest value approached in the cold stream: $M$ approaches unity at two locations, but the flow never passes through the sonic line, whereas the hot stream is always subsonic. The outcome that the sonic condition is never reached, in contrast to the onedimensional result, can be attributed to stream tube divergence, or unsteadiness, or both. As discussed in the previous section, stream tube divergence can be associated with the cross-sectional area expansion, $\delta_{a}$. Contour values of the area expansion are shown in figure $17(e)$, for two positive values of the variable. The locations of the two regions of large cross-section stretch correspond to the regions of high temperature gradient (figure 17a) and regions of high $\mathrm{OH}$ concentration (figure 17b). Moreover, the values of area expansion in these regions are comparable to those of the volume dilatation field. Therefore, figure 17(e) demonstrates a non-negligible contribution by the stream tube divergence to the dilatation where chemical activity is pronounced.

As discussed in $\S 5.1 .2$, the two other chemical systems do not support a regular combustion structure, but localized explosion fronts develop in the cold stream. This agrees with the experimental observations of Austin (2003), and large-scale computational simulations of Khokhlov et al. (2004), who showed that the explosions are associated with large localized pressure gradients. The explosions are unsteady and two-dimensional in nature, and produce shock waves in the supersonic stream that propagate upstream towards the inflow boundary. The current simulations are stopped when the shock reaches the inflow boundary. Pressure and vorticity contours for the hydrogen-oxygen-nitrogen system are shown in figure 18. The same quantities for the propane system are shown in figure 19. The thick dashed line represents the locus where the temperature has reached $90 \%$ of the burnt equilibrium value. The assumption is made that this locus roughly represents the position of the transversal ignition front as it propagates into the fresh mixture owing to molecular diffusion. Thus, a comparison between figures $18(b)$ and $19(b)$ reveals that, for the high activation energy case, the burning front has totally covered the region of high vorticity while for the hydrogen-oxygen-nitrogen system, the ignition front interacts with the fluid dynamic instability. The shape of the isothermal, thick dashed line, suggests that thermal convective transport within vortical structures is modest for the propane system when compared to the hydrogen-oxygen-nitrogen system. For both mixtures, the localized explosions form at a distance from the triple point smaller than the cold stream induction distance, cf. table 1; this is due to the effect of molecular transport.

Figures 18(a) and 19(a) also show that the explosion is much weaker in the hydrogen-oxygen-nitrogen system than in the propane system. In the latter case, the pressure shock waves spread across the boundary layer reaching the hot stream, whereas in the medium activation energy case they are confined to the cold stream and propagate one-dimensionally. Vortical structures are markedly more pronounced in the hydrogen-oxygen-nitrogen system than in the propane system. This is in agreement with the results of the linear analysis. The size of the vortical structures for the hydrogen-oxygen-nitrogen system cannot be compared to those in figure $2(b)$ for the more dilute nitrogen mixture because the formation of shocks in the cold 

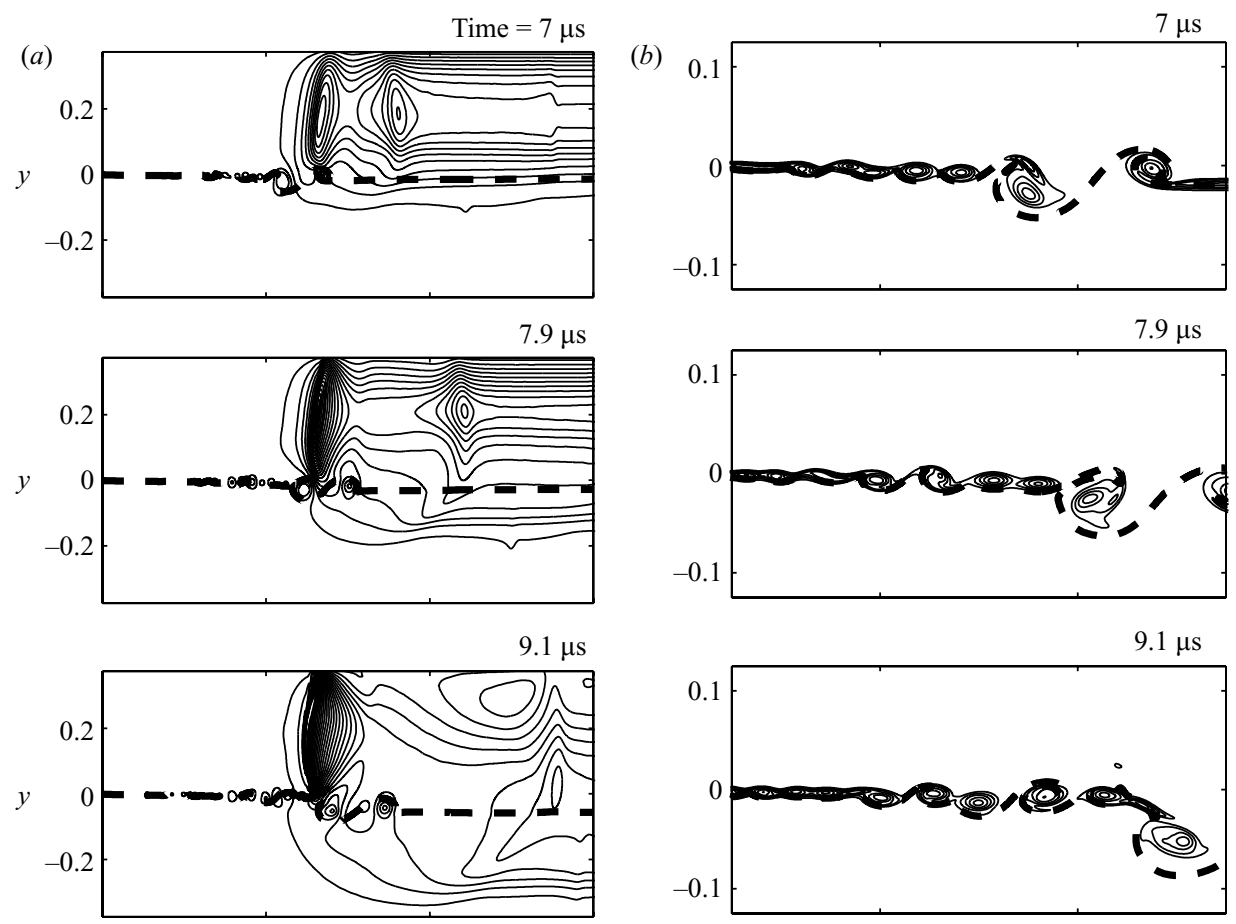

$.1 \mu \mathrm{s}$
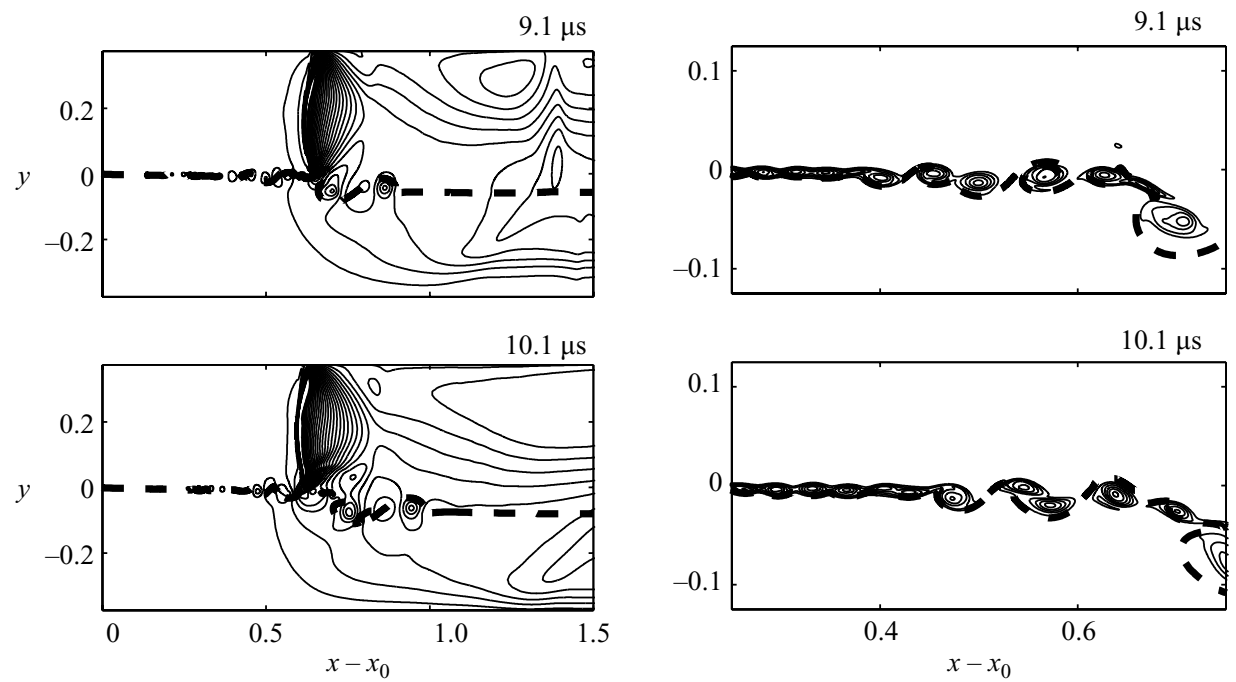

FIGURE 18. Numerical simulation of the hydrogen-oxygen-nitrogen system shear layer. The thick dashed lines represent the locus where the temperature has reached $90 \%$ of its equilibrium value. Distances are in cm. (a) Pressure, 30 contours in [0.5, 1.5] MPa. (b) Vorticity, 30 contours in $[-4,0.2] \mu \mathrm{s}^{-1}$.

stream prevents us from simulating the shear layer for a time comparable to the cell period. We note, however, that at the maximum simulated time, the formation of vortical structures at a distance of $\approx 5 \mathrm{~mm}$ from the triple point is in agreement with the experiments.

The effect of vortical structures on the combustion field is analysed through the distribution of the chemical energy release,

$$
H=\frac{\sum_{k=1}^{N_{s}} \dot{\omega}_{k}}{\rho c_{p}},
$$

where the denominator includes the local values of the density and mixture averaged specific heat. $H$ is plotted for the three cases at $7 \mu$ s in figure 20 . The plot shows that in the low and medium activation energy cases (figure $20 a, b$ ), there is a considerable 
(a)
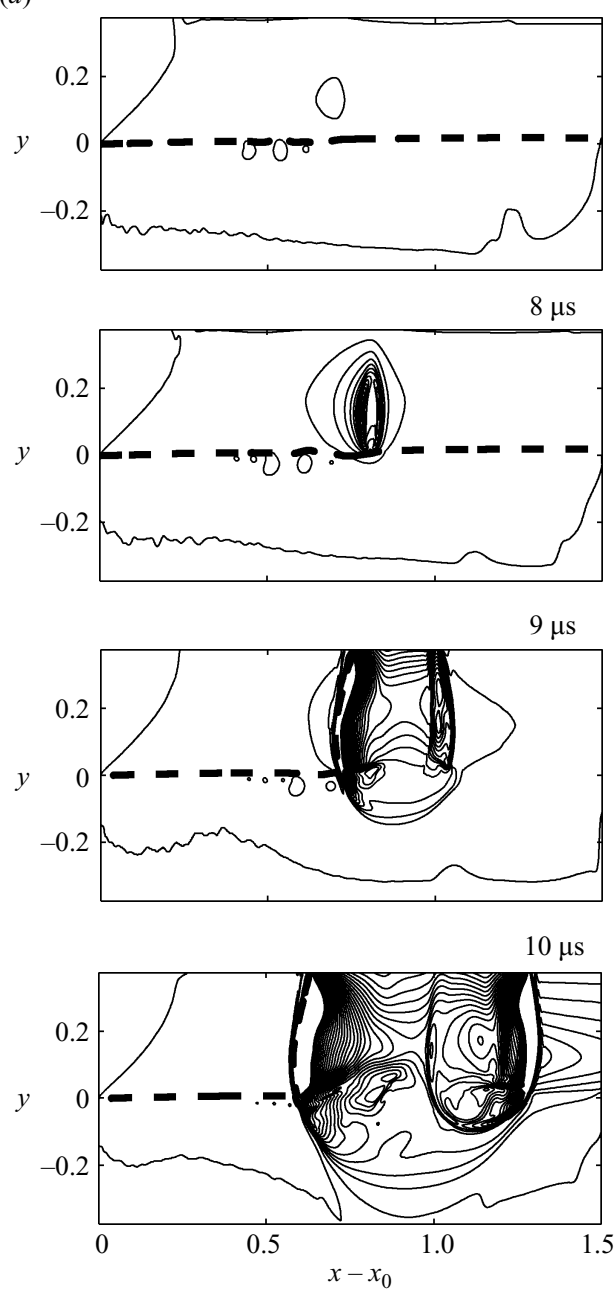

(b)
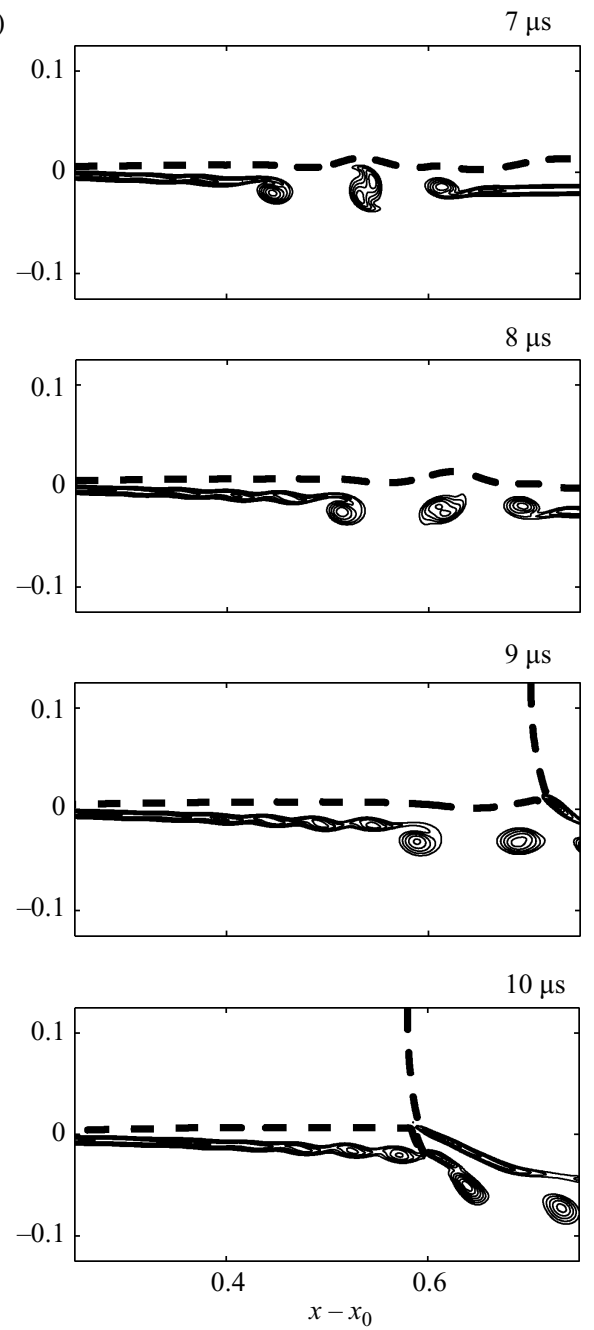

FIGURE 19. Numerical simulation of the propane system shear layer. The thick dashed lines represent the locus where the temperature has reached $90 \%$ of its equilibrium value. Distances are in $\mathrm{cm}$. (a) Pressure, 30 contours in $[0.85,3] \mathrm{MPa}$. (b) Vorticity, 30 contours in [-2, $-0.2] \mu \mathrm{s}^{-1}$.

amount of heat generated in the mixing region, whereas for the high activation energy case (figure 20c), the majority of the heat is released at the location of the transversal ignition front. The heat released in the mixing region is always lower that that generated at the front, a consequence of the premixed nature of the combustion field. The lack of heat release in the mixing region of the high activation energy case is due both to the reduced size of the vortical structures and to the high rate of consumption of fresh reactant at the (transversal) front. Thus, from the energetic standpoint, the mixing region is of greater importance for low activation energy mixtures.

The link between instability and explosions can be analysed by seeking a statistical description of convective transport by large-scale vortical structures. Figures 18 and 19 show that explosions develop near the mixing region. Local heat release enhancements due to entrainment in the mixing region are due to both temperature 
(a)

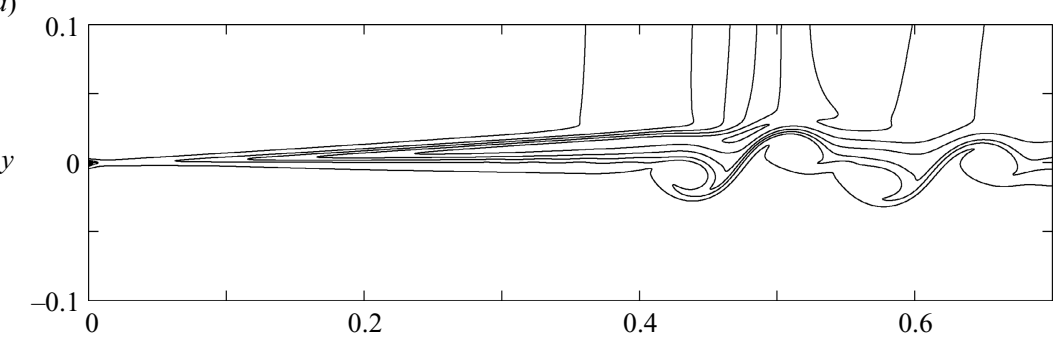

(b)

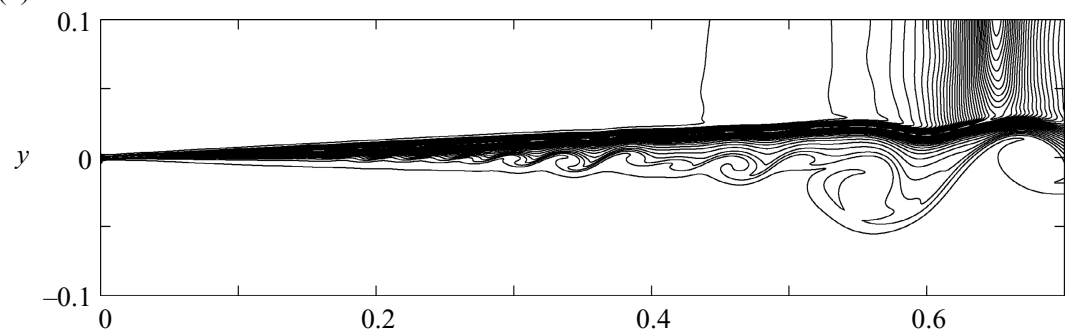

(c)

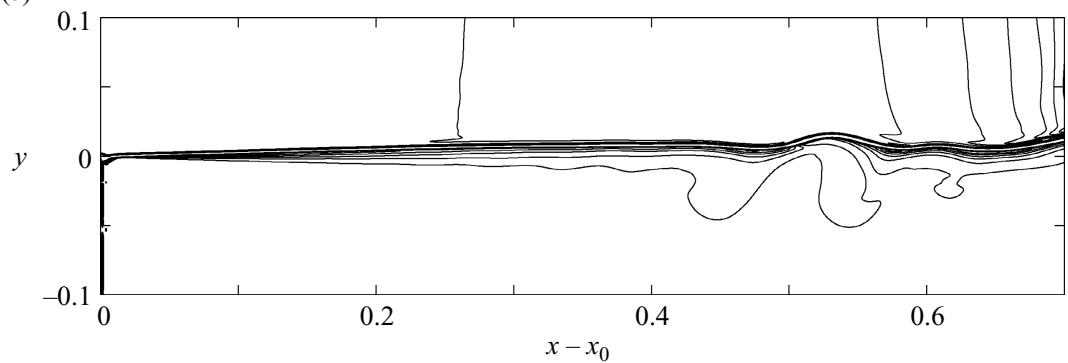

FiguRE 20. Heat release, (5.10), contours for the three systems at $7 \mu \mathrm{s}, 40$ contours in $[5,1200] \mathrm{K} \mu \mathrm{s}^{-1}$. Distances are in $\mathrm{cm}$. (a) Hydrogen-oxygen-argon, (b) hydrogen-oxygennitrogen and $(c)$ propane system.

and concentration difference between the two streams. Therefore, the quantitative analysis focuses on both the thermal and the $\mathrm{OH}$ mass fraction field.

The magnitude of the entrainment is analysed by looking at the local (pointwise) probability density function (PDF). Although the process in not stationary because of the formation of localized explosions in the medium and high activation energy mixtures, the PDF are built for the time sequences defined by the thermo-chemical history at fixed locations. The local probability density functions, $\Psi(x, y ; T)$ and $\Psi\left(x, y ; Y_{\mathrm{OH}}\right)$, are taken over the time interval $[7,10] \mu \mathrm{s}$, and the field variables are non-dimensionalized using the difference between states 3 and 4 ,

$$
\hat{T}=\frac{T-T_{3}}{T_{4}-T_{3}}
$$

an analogous formula for $Y_{\mathrm{OH}}$ defines a scaled mass fraction, $\hat{Y}$. The time interval was selected on the basis of the time frame over which ignition occurs for the three mixtures.

In this context, it is useful to provide a statistical description of entrainment by considering $80 \%$ confidence intervals, defined as the minimum interval of values of 
a random variable that contains $80 \%$ of the data. In this analysis each location in the flow field is considered separately as a random variable. More specifically, the following quantities are evaluated,

$$
\begin{gathered}
\Delta T_{80}=\min \left\{\Delta T \mid \int_{\hat{T}}^{\hat{T}+\Delta T} \Psi(x, y ; T) \mathrm{d} T>0.8\right\}, \\
\Delta Y_{\mathrm{OH}, 80}=\min \left\{\Delta Y \mid \int_{\hat{Y}}^{\hat{Y}+\Delta Y} \Psi\left(x, y ; Y_{\mathrm{OH}}\right) \mathrm{d} Y_{\mathrm{OH}}>0.8\right\} .
\end{gathered}
$$

The confidence interval is plotted versus the crosswise coordinate in figure 21 for two axial locations and for the three mixtures considered.

The width of the interval over which $\Delta T_{80}$ is non-negligible is a measure of the deviation of the solution from the one-dimensional steady structure owing to convection normal to the mean flow. Thus figures 21(e) and 21( $f$ ) show that convective fluid transport across the layer is modest in the propane system when compared to the two other systems (figure $21 a-d$ ). The temperature $80 \%$ confidence intervals for the low and medium activation energy mixtures are comparable, a result expected based upon the linear stability results of $\S 4.5$. The hydrogen-oxygen-argon system has a larger maximum value of $\Delta T_{80}$ than the hydrogen-oxygen-nitrogen system. For the two higher activation energy cases, the location of the ignition front can be identified with that of the large $\Delta Y_{\mathrm{OH}, 80}$ spike to the right of the $y=0$ abscissa in figure $21(d, f)$. This location corresponds well with that predicted on the basis of the transversal ignition analysis, cf. $\S 3.2$. For an axial distance of $\Delta x=0.5 \mathrm{~cm}$, the term $\Delta x v^{*} / u_{3}$ takes values $0.02 \mathrm{~cm}$ and $0.013 \mathrm{~cm}$ for the hydrogen-oxygen-nitrogen and propane system, respectively, which corresponds well with the location of the $\Delta Y_{\mathrm{OH}, 80}$ spike in figure $21(d, f)$. The larger predicted value for the propane system is due to the displacement of the triple-point streamline towards the negative $y$ direction as a consequence of chemical activity in the cold stream.

Large values of the confidence interval close to the ignition front are due to its fluctuation. Figures 21(c) to 21(e) confirm that thermal and concentration gradients associated with the transversal flame are comparable to those generated in the mixing region by the instability for the medium activation energy mixture, whereas they are of much smaller magnitude for the high activation energy mixture. The two local maxima of $\Delta T_{80}$ to the left of that identifying the transversal flame delimit the region of entrainment by large-scale structures. The fact that large $\Delta Y_{\mathrm{OH}, 80}$ in figure $21(c, e)$ corresponds to large $\Delta T_{80}$ values in figure $21(d, f)$ demonstrates interaction between the Kelvin-Helmholtz instability and chemical processes, i.e. vortical structures generate pockets of high reactivity. This phenomenon is pronounced in the medium mixture, whereas it is almost absent in the high activation energy mixture (figure $21 f$ ).

These concepts can be substantiated by looking at the probability density functions for the scaled temperature and $\mathrm{OH}$ mass fraction at a single location. The location of the maximum temperature confidence interval at $x-x_{0}=0.5 \mathrm{~cm}$ has been selected as representative. The PDFs for the three mixtures are shown in figure 22. The temperature PDFs (figure 22a,c,e) represent a situation typical in large-scale transport, where the PDF has maxima at the two extrema of the temperature range. The lack of observations in the middle of the temperature range points to the convective nature of the entrainment. Figures $22(b)$ and $22(d)$ show that at this location and in the time interval considered, the $\mathrm{OH}$ mass fraction is always larger 
(a)

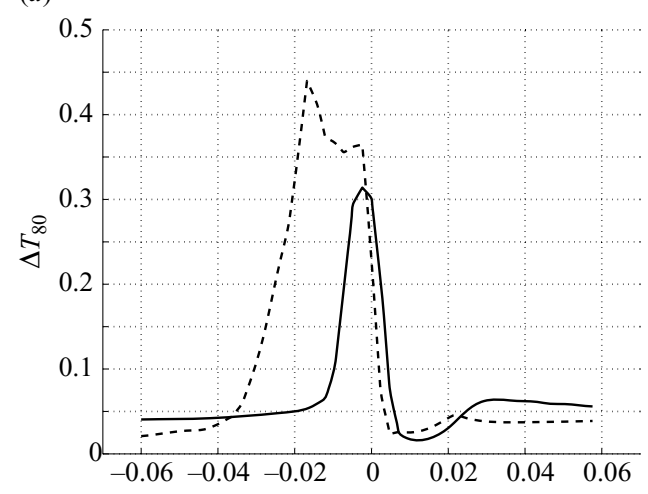

(c)

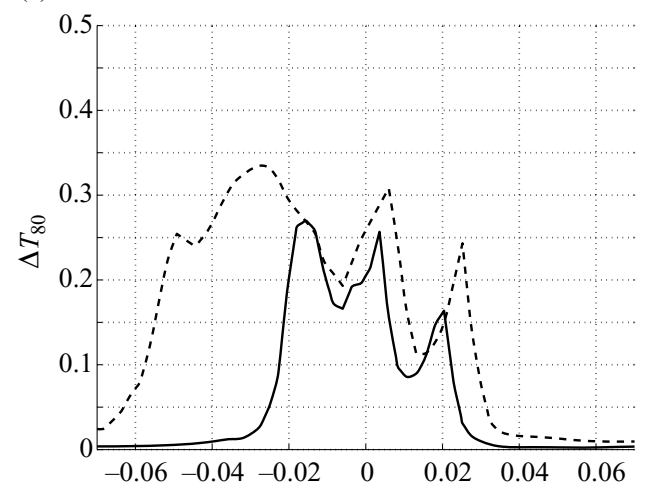

(e)

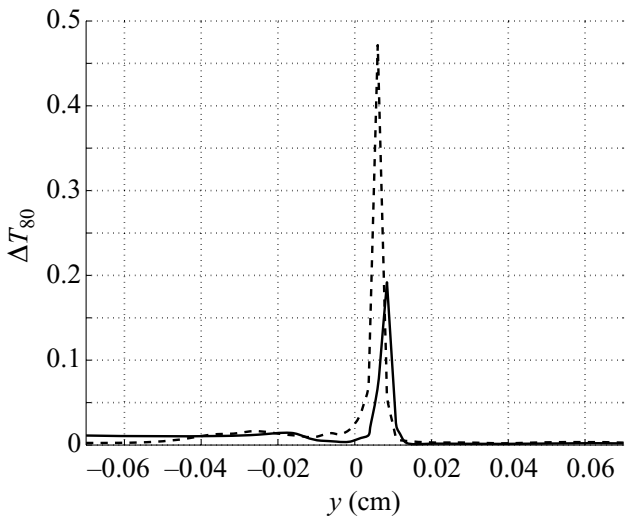

(b)

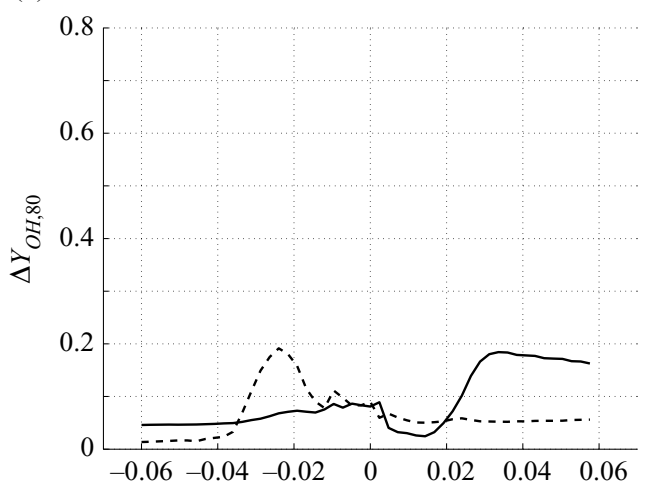

$(d)$

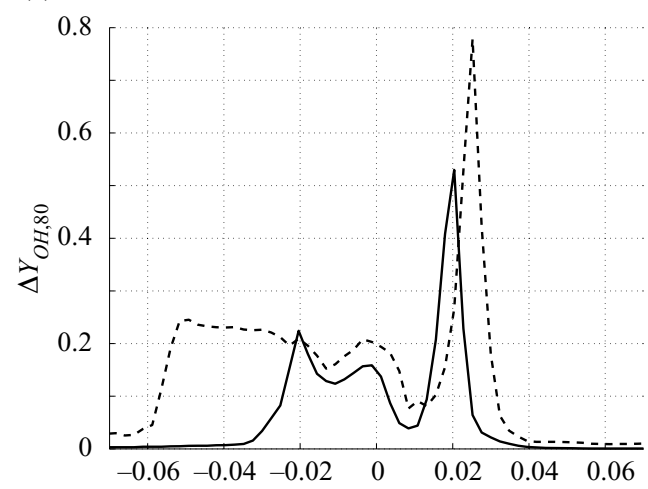

$(f)$

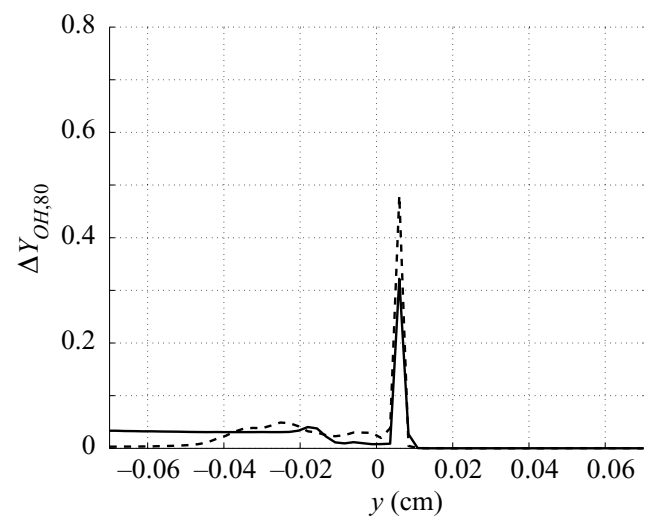

FIGURE 21. $80 \%$ confidence intervals for the scaled temperature $(a, c, e)$ and $\mathrm{OH}$ mass fraction $(b, d, f)$. The solid line is taken at $x-x_{0}=0.5 \mathrm{~cm}$ and the dashed line at $x-x_{0}=0.6 \mathrm{~cm} .(a, b)$ Hydrogen-oxygen-argon, $(c, d)$ hydrogen-oxygen-nitrogen, $(e, f)$ propane.

than the state 4 value, indicating an enhanced chemical activity in the entrainment region. The large $\mathrm{OH}$ radical concentration at the $y$ location, for which the effect of the entrainment is maximum, relates to the interaction between chemical processes and instability for the lower activation energy mixtures. Turning our attention to the propane system, we note the absence of regions with $\hat{T}>1$ or $\hat{Y}>1$ (cf. figure $22 e, f$ ). This is a consequence of the lack of mixing, which would induce reaction at higher 
(a)

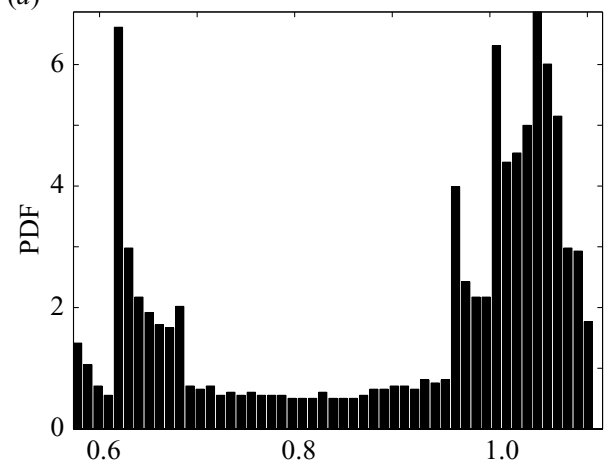

(c)

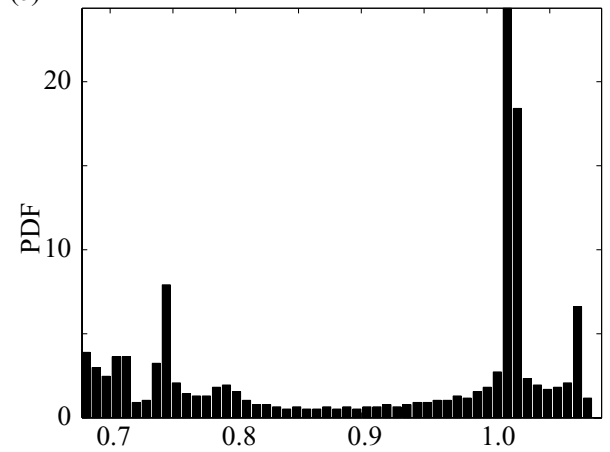

(e)

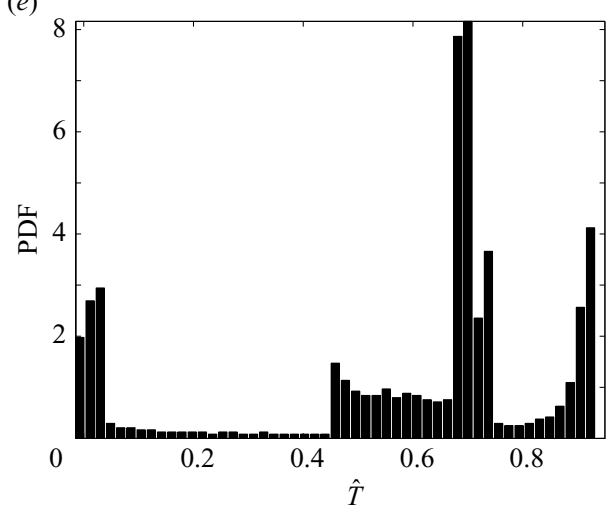

(b)

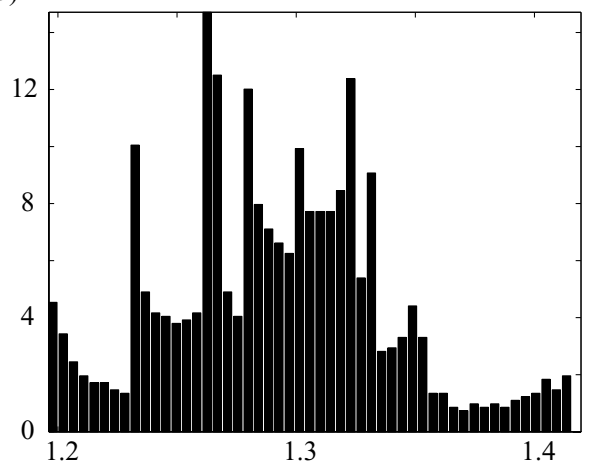

(d)

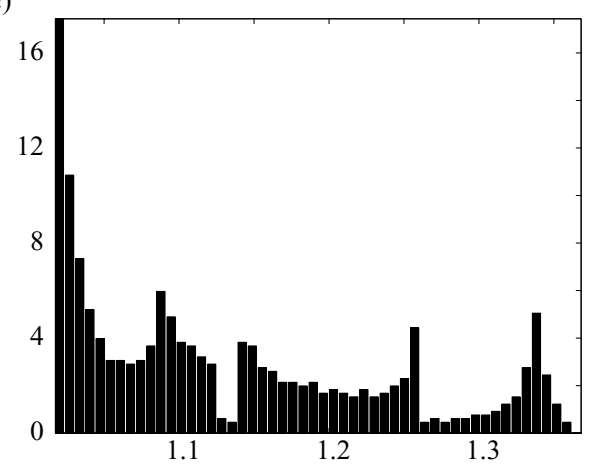

$(f)$

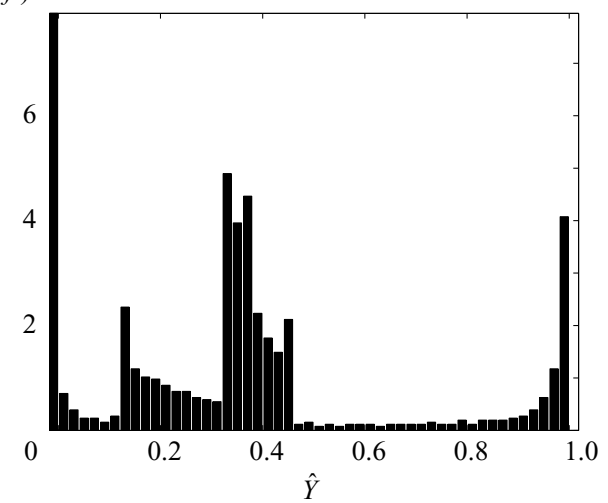

FIGURE 22. PDF of the scaled temperature $(a, c, e)$ and $\mathrm{OH}$ mass fraction $(b, d, f)$ taken at the location of the maximum temperature confidence interval for $x-x_{0}=0.5 \mathrm{~cm} .(a, b)$ Hydrogen-oxygen-argon, $(c, d)$ hydrogen-oxygen-nitrogen, $(e, f)$ propane systems.

temperatures. Therefore, time changes of the temperature field are due only to the fluctuations of the front. Also of note in figure $22(e)$ are the negative values of $\hat{T}$. These values can be explained by noting that the gas temperature falls below the cold-stream value for the hydrocarbon mixture at the edges of the mixing region, a phenomenon associated with the early stages of propane consumption, see $\S 3.1$.

In summary, the nonlinear analysis has shown that the interaction between instability structures and chemistry is of modest, if any, impact for the high activation energy system. This conclusion is explained by both the reduced instability spatial 
growth rate due to the decoupling of fluid dynamic and thermo-chemical gradients, and by the greater importance of transversal ignition for large activation energies. The thin flame front propagates past the region of high vorticity, as shown by the dotted line in figure 19(b), so that the temperature and radical concentration difference across the shear layer is reduced. The larger interaction between transversal ignition and instability in low activation energy mixtures can be linked to both the increased thickness of the flame that becomes of the same order as the vortical structures (figure $4 d$ ) and the weakening of the front (figure $4 c$ ) in the sense that the mixture does not support adiabatic transversal propagation.

\section{Conclusions}

The numerical and analytical analyses presented in this paper were motivated by experimental observations of localized, intensely luminescent regions close to the triple-point shear layers in the latter part of the cell cycle in propagating gaseous detonation waves. The intense luminescence may be associated with localized explosions that have been observed to occur near the cell cycle apex. These features were observed only in hydrocarbon mixtures with high effective activation energy. Higher activation energy mixtures exhibit an increased difference in lead shock strengths at the triple points and therefore an increased change in properties between the unreacted cold stream and the reacted hot stream on either side of the shear layer. The induction time on the cold stream side is increased, and experiments have shown Kelvin-Helmholtz-type instabilities develop between the unreacted and reacted streams. It is possible that the development of Kelvin-Helmholtz instability enhances mixing of hot products and cold reactants across the shear layers and may lead to hotspot formation and local explosions. These processes may critically augment the shock-induced thermo-chemical explosion mechanism considered to be dominant in propagating detonation fronts.

We examine the nature of the triple-point shear layers and the possible mechanisms for the appearance of localized 'hotspots' by performing (i) linear stability analysis and (ii) two-dimensional Navier-Stokes calculations with detailed chemical kinetics and transport. The shear-layer inflow conditions for the simulations are based on shock polar calculations for triple-point configurations corresponding to experimental conditions. Three mixtures have been analysed with effective activation energy in the range, $\theta_{\text {eff }} \in[5.4,12.7]$. These mixtures are chosen to correspond to the experiments.

Linear stability analysis focuses on the relation between Kelvin-Helmholtz instability and chemistry within the induction region, and analyses the spatial growth rate of the perturbations in a constant pressure mean flow. The linear stability analysis has shown that large effective activation energy mixtures have lower spatial growth-rates than low activation counterparts, and that changes in the mean profile are mostly responsible for the growth-rate attenuation. It is concluded that the decoupling between velocity gradient and thermal gradient is the mechanism underlying the growth-rate reduction. This observation establishes a correlation between activation energy and stability, but one that excludes the possibility of the localized ignition fronts being associated with an enhanced shear-layer instability for the high activation energy systems.

The set of two-dimensional Navier-Stokes simulations highlights the role of transversal ignition of the fresh mixture, a process associated with the combustion front supported by the diffusive heat transport across the shear layer. The chemical energy release at the transversal front is the dominant energetic contribution for high 
activation energy mixtures, while it is of comparable magnitude to that supported by vortical mixing for the low activation energies.

Computations predict formation of millimetre sized vortical structures in the reacted gas some distance downstream of the cold-stream auto-ignition point, for the low activation energy case. This outcome is in good agreement with the experimental studies in which vortical structures between reacted and unreacted gas have not been observed to date. For the two higher activation energy cases, localized ignition fronts are predicted to form in the non-reacted stream. These localized fronts are unsteady and two-dimensional, originate close to the transversal ignition front, and rapidly propagate through the mixture. The advection of the localized ignition fronts in the fresh mixture is through shock waves. These 'hotspots' are characterized by large pressure peaks, a temperature significantly higher than that of the constant pressure equilibrium, and a high concentration of $\mathrm{OH}$ mass fraction. All these facts are consistent with the observation of intensely luminescent regions in the experiments.

The interaction between mixture ignition and the development of the instability has been analysed by considering the entrainment of high-temperature reactive gas in the mixing region. The entrainment level is quantified on the basis of the thermal and radical transport. The simulations have shown that the level of entrainment diminishes with an increase in effective activation energy, and it is almost absent in the highest activation energy mixture. This outcome is in agreement with the linear stability analysis. The thin transversal ignition front and the decreased instability growth rate in the high effective activation energy mixture cause the reacted gas region to completely consume the high vorticity region, thus limiting entrainment. The realization that the propagation of the transversal front is diffusion controlled points to the importance of diffusion effects in detonation systems.

In conclusion, shear-layer instability appears to play no role in the formation of localized explosions. The observed 'hotspots' occur in the high and medium activation energy mixtures near the sonic-transition locus of the initially supersonic unreacted stream. This outcome is explained by noticing that the unreacted stream does not support a one-dimensional equilibrium solution that preserves the injection values of mass flow rate, momentum flux, total enthalpy and atomic fluxes in the lead shock reference frame. The existence of a one-dimensional equilibrium is a necessary condition for the existence of a steady reactive wave and for the existence of a statistically-stationary premixed combustion shear-layer solution when viscous effects and stream tube expansion are not important. The low activation energy system supports a regular solution devoid of localized ignition fronts, even though equilibrium one-dimensional states do not exist in the natural shock frame; the stream-tube crosssectional area expansion is the key factor in the stabilization of the flow. The reason why the larger effective activation energy mixtures are not stabilized by streamtube expansion is probably related to the much faster volume build-up at ignition. Molecular diffusion heating is responsible for the fact that thermal runaway initiates close to the transversal ignition front, a conclusion that explains the localized and multidimensional nature of the explosions.

L.M. and T.L.J. were supported by the US Department of Energy through the University of California under subcontract number B523819.

\section{REFERENCES}

Arienti, M. \& Shepherd, J. E. 2005 The role of diffusion at shear layers in irregular detonations. Joint Meeting of the US Sections of the Combustion Institute, Philadelphia, PA. 
Austin, J. M. 2003 The role of instability in gaseous detonation. PhD thesis, California Institute of Technology, Pasadena, California.

Austin, J. M., Pintgen, F. \& Shepherd, J. E. 2005a Lead shock oscillation and decoupling in propagating detonations. AIAA Paper 2005-1170.

Austin, J. M., Pintgen, F. \& Shepherd, J. E. $2005 b$ Reaction zones in highly unstable detonations. Proc. Combust. Instute 30, 1849-1857.

Buckmaster, J. D. \& Ludford, G. S. S. 1982 Theory of Laminar Flames. Cambridge University Press.

Criminale, W. O., Jackson, T. L. \& Joslin, R. D. 2003 Theory and Computation of Hydrodynamic Stability. Cambridge University Press.

Day, M. J., Reynolds, W. C. \& Mansour, N. N. 1998 The structure of the compressible reacting mixing layer: insights from linear stability analysis. Phys. Fluids 10 (4), 993-1007.

DiXon-Lewis, G. 1968 Flame structure and flame reaction kinetics. II. Transport phenomena in multicomponent systems. Proc. R. Soc. Lond. A 307, 111-135.

Edwards, D. H., Hooper, G. \& Meddins, R. J. 1972 Instabilities in the reaction zones of detonation waves. Astronaut. Acta 17, 475-485.

Erpenbeck, J. J. 1964 Stability of idealized one-reaction detonations. Phys. Fluids 7, 684-696.

Gamezo, V. N., Desbordes, D. \& Oran, E. S. 1999a Formation and evolution of two-dimensional cellular detonations. Combust. Flame 116, 154-165.

Gamezo, V. N., Desbordes, D. \& Oran, E. S. $1999 b$ Two-dimensional reactive flow dynamics in cellular detonation waves. Shock Waves 9, 11-17.

Gamezo, V. N., Vasilev, A. A., Khokhlov, A. M. \& Oran, E. S. 2000 Fine cellular structures produced by marginal detonations. In 28th Symp. (Intl) on Combustion, pp. 611-617. The Combustion Institute.

Hermanson, J. C. \& Dimotakis, P. E. 1989 Effects of heat release on a turbulent, reacting shear layer. J. Fluid Mech. 199, 333-375.

JACKSON, T. L. \& GROSCH, C. E. 1989 Inviscid spatial stability of a compressible mixing layer. J. Fluid Mech. 208, 609-637.

JACKSON, T. L. \& GrosCh, C. E. 1990 Absolute/convective instability and the convective Mach number in a compressible shear layer. Phys. Fluids 2, 949-954.

JACKSON, T. L. \& GrosCH, C. E. 1991 Inviscid spatial stability of a compressible mixing layer. Part 3. Effect of thermodynamics. J. Fluid Mech. 224, 159-175.

Kee, R. J., Dixon-Lewis, G., Warnatz, J., Coltrin, M. E. \& Miller, J. A. 1986 A FOrTran computer code package for the evaluation of gas-phase multicomponent transport properties. Rep. SAND86-8246. Sandia National Laboratories.

Kee, R. J., Rupley, F. M., Meeks, E. \& Miller, J. A. 1996 CHEMKin-III: A FORTRAN chemical kinetics package for the analysis of gasphase chemical and plasma kinetic. Rep. SAND96-8216. Sandia National Laboratories.

Kennedy, C. A. \& CARPENTER, M. H. 1994 Several new numerical methods for compressible shear layer simulations. Appl. Numer. Math. 14, 397-433.

KenNedy, C. A. \& CARPENTER, M. H. 1997 Comparison of several numerical methods for simulation of compressible shear layers. NASA TP 3484.

Khokhlov, A. M., Austin, J. M., Pintgen, F. \& Shepherd, J. E. 2004 Numerical study of the detonation wave in ethylene-oxygen mixtures. AIAA Paper 2004-0792.

Konnov, A. A. 2000 Development and validation of a detailed reaction mechanism for the combustion of small hydrocarbons. In 28th Symp. (Intl) on Combustion, p. 317. The Combustion Institute.

LeE, H. I. \& Stewart, D. S. 1990 Calculation of linear detonation instability: one-dimensional instability of plane detonation. J. Fluid Mech. 216, 103-132.

Lock, R. C. 1951 The velocity distribution on the laminar boundary layer between parallel streams. Q. J. Mech. Appl. Maths 4, 42-63.

Lundstrom, E. A. \& Oppenheim, A. K. 1969 On the influence of non-steadiness on the thickness of the detonation wave. Proc. R. Soc. Lond. A 310, 463-478.

Oppenheim, A. K., Smolen, J. J. \& ZaJAC, L. J. 1968 Vector polar method for the analysis of wave intersections. Combust. Flame 12, 63-76.

Pintgen, F., Austin, J. M. \& Shepherd, J. E. 2003 a Confined detonations and pulse detonation engines. In Detonation Front Structure: Variety and Characterization (ed. G. D. Roy, S. M. Frolov, R. J. Santoro \& S. A. Tsyganov). Torus, Moscow. 
Pintgen, F., Eckett, C. A., Austin, J. M. \& Shepherd, J. E. $2003 b$ Direct observations of reaction zone structure in propagating detonations. Combust. Flame 133, 211-229.

Poinsot, T. J. \& Lele, S. K. 1992 Boundary conditions for direct simulations of compressible viscous flows. J. Comput. Phys. 101, 104-129.

Rosenhead, L. (ed.) 1963 Laminar Boundary Layers. Oxford University Press.

SCHultz, E. \& ShePherd, J. E. 2000 Validation of detailed reaction mechanisms for detonation simulation. Tech. Rep. FM99-5. Graduate Aeronautical Laboratories: California Institute of Technology.

Shampine, L. F., Gladwell, I. \& Brankin, R. W. 1991 Reliable solutions of special event location problems for ODEs. ACM Trans. Math. Software 17 (1), 11-25, available @ http://www.netlib.org.

Shin, D. \& Ferziger, J. 1993 Linear stability of the compressible reacting mixing layer. AIAA J. 31, 677-685.

Short, M. \& Quirk, J. J. 1997 On the nonlinear stability and detonability limit of a detonation wave for a model three-step chain-branching reaction. J. Fluid Mech. 339, 89-119.

Short, M. \& Stewart, D. S. 1998 Cellular detonation stability. Part 1. A normal-mode linear analysis. J. Fluid Mech. 368, 229-262.

Singh, S., Leiberman, D. \& Shepherd, J. E. 2003 Combustion behind shock waves. In Western States Section. The Combustion Institute. Paper 03F-29.

Smith, G. P., Golden, D. M., Frenklach, M., Moriarty, N. W., Eiteneer, B., et al. 2000 GRI-Mech 3.0. http://www.me.berkeley.edu/gri_mech/.

Strehlow, R. A. 1969 The natue of transverse waves in detonations. Astronaut. Acta 14, 539-548.

Strehlow, R. A. \& CROOKER, A. J. 1974 The structure of marginal detonation waves. Acta Astronaut. 1, 303-315.

Subbotin, V. A. 1975 Two kinds of transverse wave structures in multi-front detonation. Fizika Goreniya i Vzryva 11 (1), 96-102.

Takai, R., Yoneda, K. \& Hikita, T. 1974 Study of detonation wave structure. In 15th Symp. (International) on Combustion, pp. 69-78. Pittsburgh, PA: The Combustion Institute.

Urtiew, P. A. 1970 Reflections of wave intersections in marginal detonations. Astronaut. Acta 15, 335-343.

Vasiliev, A. A. \& Nikolaev, Yu. 1978 Closed theoretical model of a detonation cell. Acta Astronaut. 5, 983-996.

Voitsekhovski,, B. V., Mitrofanov, V. V. \& Topchian, M. E. 1963 Struktura fronta detonastii i gaza. Akad. Nauk. SSSR, Novosibirsk. Translation: The structure of a detonation front in gases. Rep. FTD-MT-64-527, Foreign Technology Division, Wright-Patterson A.F.B., Ohio (1966).

White, D. R. 1961 Turbulent structure of gaseous detonation. Phys. Fluids 4, 465-480. 